\title{
Public Discourse and Social Network Echo Chambers Driven by Socio-Cognitive Biases
}

\author{
Xin Wang $\odot,{ }^{1,2}$ Antonio D. Sirianni $\odot,{ }^{3, *}$ Shaoting Tang, ${ }^{1}$ Zhiming Zheng, ${ }^{1}$ and Feng Fu $\odot^{2,4, \dagger}$ \\ ${ }^{1} L M I B, S K L S D E, B D B C, P C L$ and School of Mathematical Sciences, Beihang University, \\ Beijing 100191, China \\ ${ }^{2}$ Department of Mathematics, Dartmouth College, Hanover, New Hampshire 03755, USA \\ ${ }^{3}$ Department of Sociology, Dartmouth College, Hanover, New Hampshire 03755, USA \\ ${ }^{4}$ Department of Biomedical Data Science, Dartmouth College, Lebanon, New Hampshire 03756, USA
}

(Received 6 June 2020; revised 1 September 2020; accepted 27 October 2020; published 1 December 2020)

\begin{abstract}
In recent years, social media has become an important platform for political discourse, being a site of both political conversations between voters and political advertisements from campaigns. While their individual influences on public discourse are well documented, the interplay between individual-level cognitive biases, social influence processes, dueling campaign efforts, and social media platforms remains unexamined. We introduce an agent-based model that integrates these dynamics and illustrates how their combination can lead to the formation of echo chambers. We find that the range of political viewpoints that individuals are willing to consider is a key determinant in the formation of polarized networks and the emergence of echo chambers and show that aggressive political campaigns can have counterproductive outcomes by radicalizing supporters and alienating moderates. Our model results demonstrate how certain elements of public discourse and political polarization can be understood as the result of an interactive process of shifting individual opinions, evolving social networks, and political campaigns. We also introduce a dynamic empirical case, retweet networks from the final stage of the 2016 U.S. presidential election, to show how our proposed model can be calibrated with real-world behavior.

DOI: $10.1103 /$ PhysRevX.10.041042

Subject Areas: Complex Systems, Interdisciplinary Physics
\end{abstract}

\section{INTRODUCTION}

People are more likely to accept claims that are coherent with their preexisting beliefs [1]. Furthermore, people seek out individuals with similar beliefs. These two mechanisms, confirmation bias and selective exposure [2,3], can generate polarized and homogeneous clusters of opinion, commonly referred to as echo chambers [4]. Echo-chamber effects may stop individuals from being exposed to information or opinions contrary to their extant beliefs and further radicalize individuals with extreme beliefs [5]. Political polarization in the United States has increased markedly in recent decades [6]. While confirmation bias and selective exposure are not necessarily new phenomena, new digital environments have arisen that can accelerate echo-chamber emergence and then entrench them in the public discourse. Making matters more complicated, political campaigns may aim to strategically

\footnotetext{
*antonio.d.sirianni@dartmouth.edu

${ }^{\dagger}$ Feng.Fu@dartmouth.edu
}

Published by the American Physical Society under the terms of the Creative Commons Attribution 4.0 International license. Further distribution of this work must maintain attribution to the author(s) and the published article's title, journal citation, and DOI. capitalize on these mechanisms and environments. Comprehensively understanding the emergence of echo chambers and large-scale political polarization in the real world requires a model of collective decision making that integrates the socio-cognitive biases that underlie individual political decisions, the digital ecologies and network structures that facilitate communication, and the strategic efforts of political campaigns.

Information consumption and diffusion has radically changed with the development of large-scale social networks $[7,8]$. News outlets are integrated with active online social networks and can now spread information faster, more widely, and more effectively $[9,10]$. Furthermore, social network users are no longer just passive recipients of information but can also create and distribute content, selectively disseminate information, and share their own opinions [11]. These changes have had a substantial impact on public discourse and opinion formation: Individuals can easily find evidence that supports their existing ideas and follow those people who hold similar viewpoints, amplifying preexisting socio-cognitive biases $[12,13]$. Recommendation algorithms further amplify these biases by predicting and delivering the content that individuals are most likely to consume $[14,15]$.

The seemingly ubiquitous formation of echo chambers on social networks has aroused concern in several different 
fields [16-18]. Sociologists and political scientists are concerned that social polarization caused by politically motivated selective exposure threatens democracies [19]. Twitter data show that political user groups are more likely to retweet users belonging to their own group and that supporters of political parties will rarely interact with rival party supporters $[20,21]$. The role of opinion-based confirmation bias in the diffusion of rumors and fake news has also attracted the attention of network and data scientists [22-25], and several empirical studies focus on social network user content consumption patterns in the age of misinformation [26-30]. Echo-chamber effects can reinforce the diffusion of rumors and intensify the segregation of their believers [31].

In addition to the extensive empirical work documenting instances of echo-chamber formation and simulation-based work that demonstrates the mechanisms sufficient for political (and cultural) polarization [32,33], public opinion evolution has also been studied via various mathematical models, such as Friedkin and Johnsen's social influence model [34-37], Axelrod's model of cultural dissemination [38], Deffuant's bounded confidence model [39,40], and others that considers the coevolution of network structure and individual beliefs $[32,41]$.
While previous work focuses on interpersonal opinion interactions and how networks evolve in response to peerinfluence processes $[42,43]$, few attempts have been made to unveil the non-negligible impacts of external political campaigns on peer-influence processes (see details in Appendixes D and E). To this end, we develop an agentbased model which explicitly incorporates top-down political campaign dynamics (including ideological positions and campaign efforts), socio-cognitive biases, and network structure to understand the formation of echo chambers and their influence on the public discourse. First, as a motivating example of political polarization, we depict the evolution of echo chambers in Twitter retweet networks during a series of major political events (debates between candidates in the 2016 U.S. presidential election) [44]. We then present our model of evolving political opinion, which not only qualitatively replicates the emergent echo-chamber formation like the kind seen in 2016, but also illustrates several interesting facets of how processes of campaign influence and peer influence interact. Our results show that polarized and segregated network structures are largely a function of the ideological difference between campaigns and the extent to which individuals are open to considering (a)

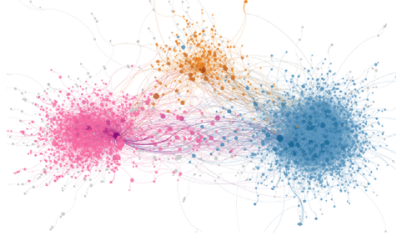

Sep. $27,00: 50$
The 2016 U.S. first presidential debate

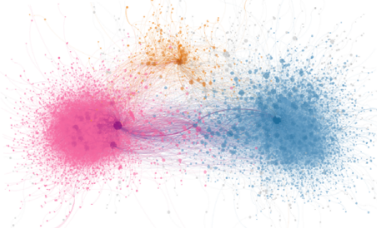

Sep. 27, 7:55

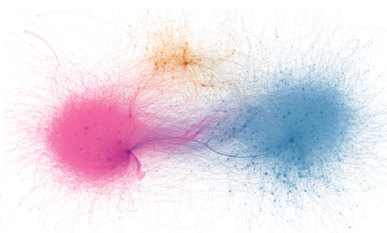

Sep. $27,15: 00$

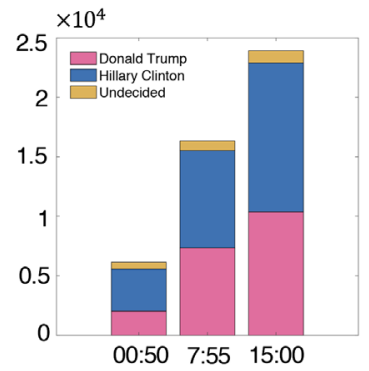

$00.50 \quad 7.55 \quad 15 \cdot 00$ (b)

The 2016 U.S. vice-presidential debate

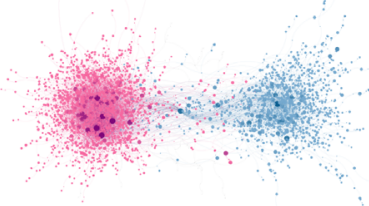

Oct. $6,14: 23$

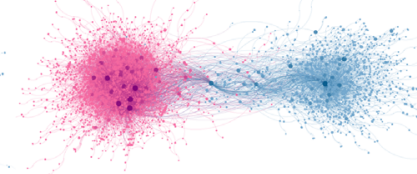

Oct. $8,13: 07$

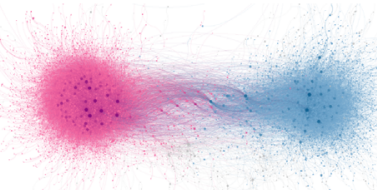

Oct. $10,11: 51$

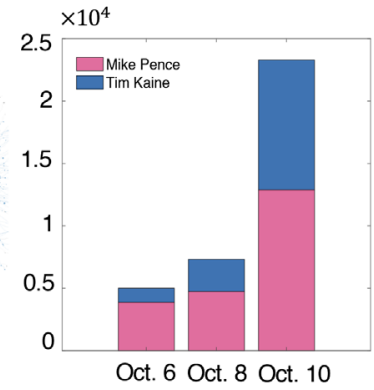

FIG. 1. Evolving echo chambers in core retweet networks. The progression of echo-chamber formation in retweet networks during time frames surrounding (a) the first 2016 U.S. presidential debate and (b) the 2016 U.S. vice presidential debate. In each network subfigure on the left, the nodes represent users, node colors show the nodes cluster, the edges indicate retweets, and node size and color intensity reflect a node's retweet volume. The bar charts on the right show the growth of support for each candidate during two of the 2016 debates. In (a), the red nodes indicate individuals who tend to support Donald Trump, while blue ones indicate Hillary Clinton supporters. The yellow cluster shows users who are mainly retweeting unaligned users or supporters of both sides. The dataset begins at 17:45 on September 26, and the final graph includes 54840 edges. In (b), the networks are divided into two polarized echo chambers: The red cluster shows Mike Pence (Trump's vice president) supporters, and the blue cluster shows Tim Kaine (Clinton's vice president) supporters. Unlike the first presidential debate, there is not a cluster of undecided supporters, despite both graphs being roughly the same size in terms of nodes and edges. The dataset begins at 15:40 on October 4, 2016, and the final graph includes 55008 edges. 
alternative viewpoints (open-mindedness). Our model also examines how confirmation and selection bias, known generators of echo chambers [31,45], influence the coevolution of political opinions and network structure. We also find, not surprisingly, that the success of political campaigns is determined both by relative campaign effort (whose voice is louder) and, more subtly, by the divergence of ideological positioning between opposing campaigns. Our model has several surprising and interesting theoretical findings. The first is that political campaigns might form echo chambers that turn off moderates by radicalizing their base. The second is that moderate levels of open-mindedness, where individuals consider different, but not too different, political opinions, are most conducive to bipartisan polarization. Finally, as an illustrative example, we demonstrate how our motivating empirical case, polarization on Twitter during the later stages of the 2016 U.S. presidential campaign, might be expressed in terms of our proposed model.

\section{RESULTS}

As a motivating example for our agent-based model, we first examine the coevolution of political opinion and retweet networks on Twitter during the final stages of the 2016 presidential campaign. We focus specifically on two highly visible political events, which are shown in Fig. 1: the first U.S. presidential debate in 2016 between Hilary Clinton and Donald Trump and the lone vice-presidential debate between Tim Kaine and Michael Pence (see Appendix A for dataprocessing details). During the first U.S. presidential debate, Twitter users form three distinct clusters: one supporting Clinton (blue), one Trump (red), and a third cluster of seemingly undecided users in the middle (yellow) [Fig. 1(a)]. The middle cluster becomes relatively smaller

\section{(a)}

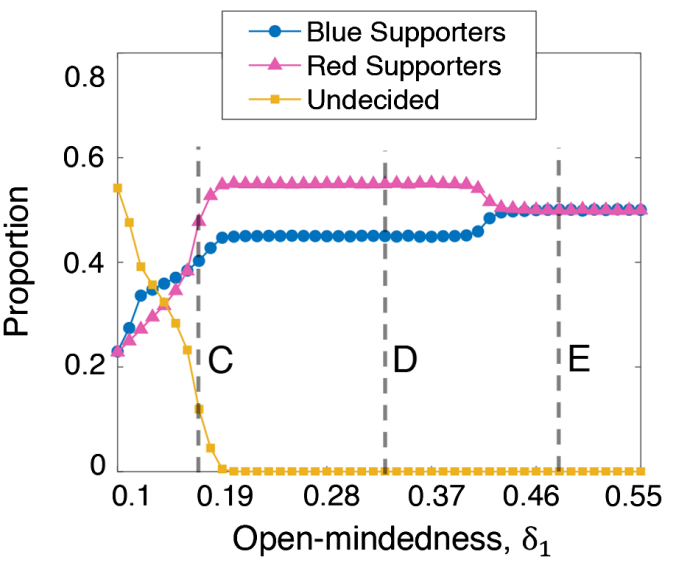

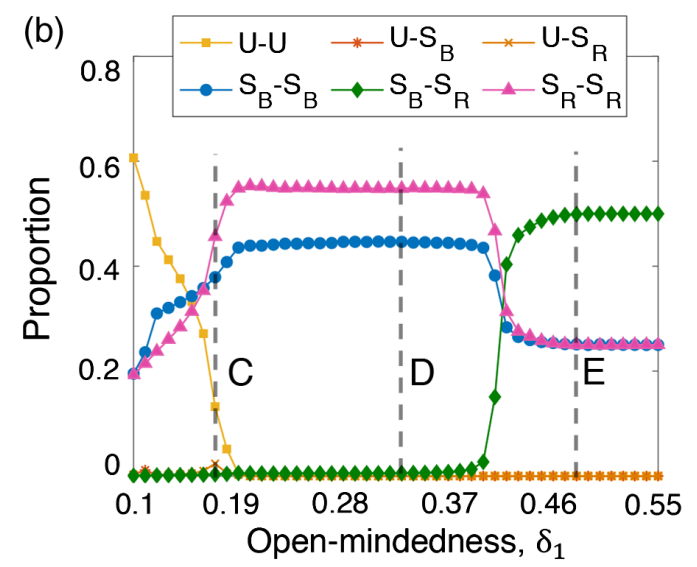

(e) One cluster, $\delta_{1}=0.48$ (c) Three clusters, $\delta_{1}=0.17$

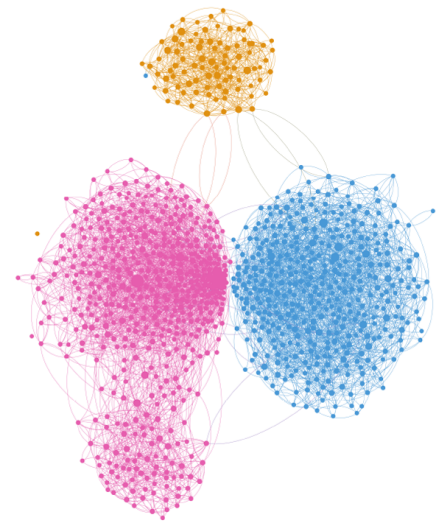

(d) Two clusters, $\delta_{1}=0.33$

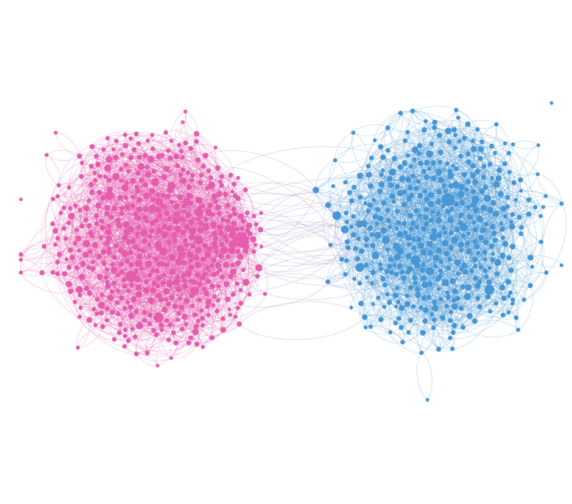

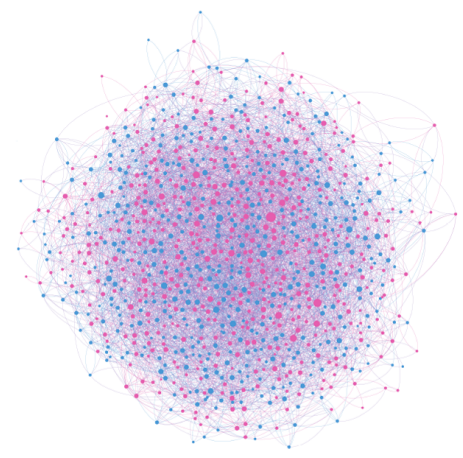

FIG. 2. Modeling the emergence of echo chambers. The relationship between the degree of ideological segregation and population identity scope (i.e., open-mindedness) largely influences the nature and outcome of public discourse. Shifts in identity scope are alone sufficient to drastically change the (a) breakdown of candidate support and (b) the distribution of ties between and within supporter groups. The stable network structures of simulations corresponding to three different identity scope values (c)-(e) show how openmindedness effects the emergence of echo chambers. Parameters: Campaign effort $\Omega_{T}=0.5$, position of viewpoint blue $\theta_{1}=0.2$, position of viewpoint red $\theta_{2}=0.7$, influence of candidate $\mu_{1}=0.5$, and influence of discussion partner $\mu_{2}=0.5$. All simulations begin from an Erdös-Rényi (ER) random graph; the simulations for (a) and (b) have network size $N=10^{4}$ and average degree $\langle k\rangle=6$. Simulation results are averaged over 100 independent runs. In the simulations that generate (c) $-(\mathrm{e}), N=10^{3}$ and $\langle k\rangle=6$. 
over time as a higher percentage of users becomes absorbed in the two extremal clusters. The Twitter snapshots surrounding the vice-presidential debate 11 days later reflect an even more polarized electorate [Fig. 1(b)]. There are only two main clusters of supporters, one for Kaine (Clinton's vice president candidate) and one for Pence (Trump's vice president candidate), that become more distinct and polarized over time. In both cases, there are only a handful of links between opposing echo chambers and dense connections within each cluster.

\section{A. Agent-based model of public discourse and echo-chamber formation}

We now introduce an agent-based model that can reproduce the patterns of dynamic polarization observed in the core retweet networks (see Fig. S1 for model parameters [46]), while accounting for the relative effort and ideological position of competing political campaigns. Consider a network $G$ with $N$ users. While several different opinions may be expressed by political and media elites, we assume without loss of generality that there are two: viewpoint blue $\left(\theta_{1}\right)$ and viewpoint red $\left(\theta_{2}\right)$, that each reflect the viewpoint of two opposing political campaigns. Each point exists on an ideological spectrum that stretches from 0 to $1\left(\theta_{i} \in[0,1]\right)$, and $\delta_{0}=\left|\theta_{1}-\theta_{2}\right|$ indicates the degree of ideological separation between the two viewpoints. Each agent $i$ has an initial belief $w_{i} \in[0,1]$ that is distributed with uniform probability, and each agent begins as undecided in the race between candidate blue and candidate red. At each time step, an individual considers updating their support to one of the two candidates, with a probability that reflects the relative effort exerted by each campaign $\left(\Omega_{T}\right)$. Their likelihood of accepting updates to their beliefs (on a 0 to 1 scale) depends on their openmindedness $\left(\delta_{1}\right)$, a constant for all individuals in the model, and the proximity of the candidate's ideology to the individual supporter's current ideology. Individuals are also connected to others in the population. If a selected agent does not accept a candidate, they will have a conversation with one of their connections and update their beliefs if their connection is ideologically close or rewire their connection if they are ideologically distant.

Specifically, the model goes through the following steps at each time step $T$ :

(1) An agent $i$ is randomly selected.

(2) Agent $i$ considers updating to either $\theta_{1}$ or $\theta_{2}$ with probability $\Omega_{T}$ and $1-\Omega_{T}$, respectively.

(3) Assume $\theta_{1}$ is selected. One of three possible interactions occur:

(a) Support candidate.-If $\left|w_{i}-\theta_{1}\right|<\delta_{1}$, then $i$ becomes a blue supporter. $i$ also becomes more ideologically similar to their new candidate, and $w_{i}$ is updated to $\bar{w}_{i}$ in accordance with a feedback parameter (candidate influence) $\mu_{1}$ : $\bar{w}_{i}=\left(1-\mu_{1}\right) w_{i}+\mu_{1} \theta_{1}, \mu_{1} \in[0,1][39]$.
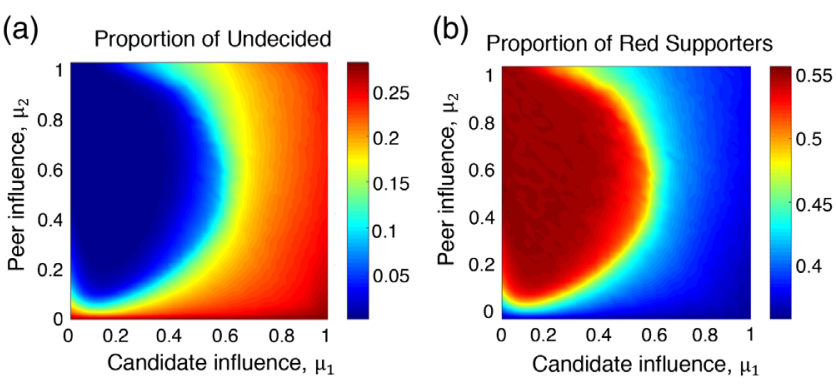

(c)

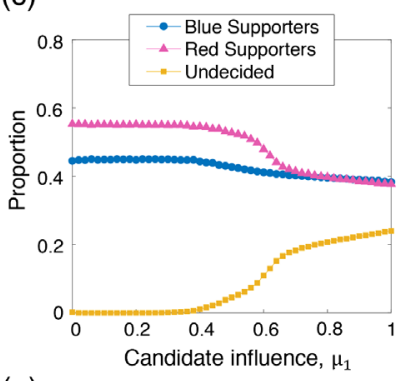

(e)
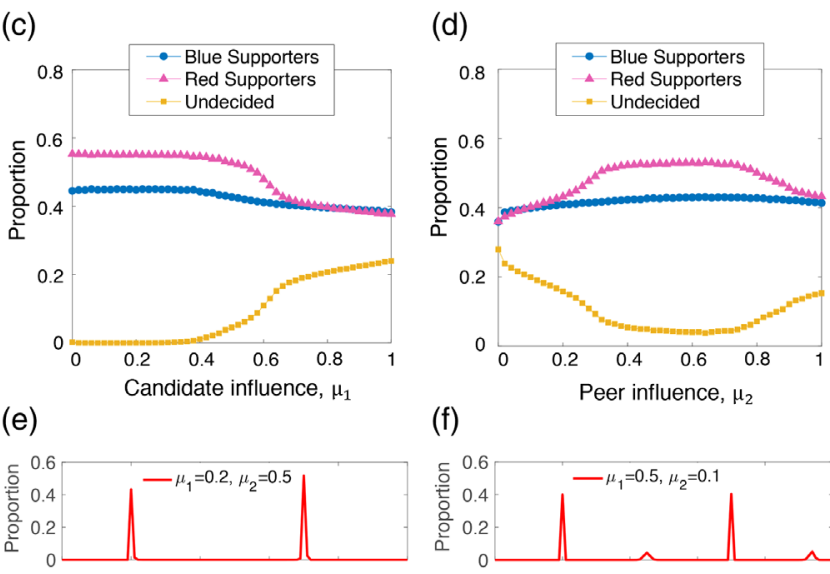

(f)

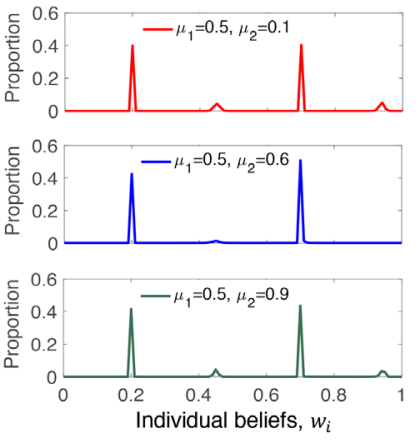

FIG. 3. Confirmation bias and attitude evolution. (a),(b) Generally, moderate discussion partner influence (peer influence) and weak candidate influence lead to larger echo chambers, in this case a significantly higher proportion of red supporters and a lower proportion of undecided individuals. (c) Strong levels of candidate influence may increase the belief distance between supporters and undecided people and accelerate opinion polarization, which hinders sufficient social discourse and results in smaller echo chambers around candidates. (d) When candidate influence is fixed, moderate levels of discussion partner influence promote more social discourse, which reduces the size of the undecided population and effectively prevents the formation of small clusters that hold extreme opinions [also see (f)]. (e),(f) Distribution of individual beliefs in steady states, corresponding to the various situations in (c) and (d). Parameters: All simulations begin from an ER graph with $N=10^{4},\langle k\rangle=6$, $\Omega_{T}=0.5, \theta_{1}=0.2, \theta_{2}=0.7$, and population open-mindedness $\delta_{1}=0.18$. In addition, (c) $\mu_{2}=0.5$ and (d) $\mu_{1}=0.5$. Simulation results are averaged over 100 independent runs.

(b) Discuss with a friend.-If $\left|w_{i}-\theta_{1}\right| \geq \delta_{1}$, then agent $i$ turns has an ideological conversation with $j$. If $\left|w_{i}-w_{j}\right|<\delta_{1}, i$ is persuaded by $j$ and updates their beliefs in accordance with a 
homogeneity parameter (peer influence) $\mu_{2}$ : $\bar{w}_{i}=\left(1-\mu_{2}\right) w_{i}+\mu_{2} w_{j}, \mu_{2} \in[0,1][47]$.

(c) Find a new friend.-If $\left|w_{i}-\theta_{1}\right| \geq \delta_{1}$ and $\left|w_{i}-w_{j}\right| \geq \delta_{1}$ for the chosen candidate and social connection, then $i$ rewires their tie with connection $j$ to another tie, $k$, where $\left|w_{i}-w_{k}\right|<\delta_{1}$.

(4) Repeat steps 1-3 until all agents update once.

The model then updates to time step $T+1$, which continues when the beliefs of individual agents have all stabilized or when the system as a whole reaches a dynamic equilibrium, which occurs when individuals collectively oscillate between supporting different candidates at a constant rate [e.g., Fig. 2(e)].

This model synthesizes the socio-cognitive biases of the individuals, the evolving structure of the social network, and the relative effort and ideological positions of campaigns. As we shift these parameters from simulation to simulation, we can look at the effect that these interacting mechanisms have on the emergence of distinct ideological clusters (echo chambers). Of particular interest is how open-mindedness can influence echo-chamber formation.
A population of agreeable people may be particularly prone to the influence of candidates, while a population of closedminded people may seek out and listen to only ideologically similar connections.

Figure 2 illustrates how the emergence of echo chambers is fundamentally related to the open-mindedness of individual voters. In this series of agent-based models, we hold the two viewpoints of candidates constant $\left(\theta_{1}=0.2\right.$ and $\left.\theta_{2}=0.7\right)$ and assume that campaign efforts are equal $\left(\Omega_{T}=0.5\right)$. Candidates and connections both have intermediate levels of influence on individual agents $\left(\mu_{1}=0.5\right.$ and $\mu_{2}=0.5$ ). As the open-mindedness (or identity scope) of individual agents in the population becomes larger, the tendency for clusters and for candidates to support changes. There are two phase shifts that occur at 0.19 and 0.43 in this series of models. At low values $\left(\delta_{1}<0.19\right)$, individuals tend to form smaller distinct ideological clusters, but these clusters may not all align around a candidate. In the case presented, there is only one cluster that is not aligned with a candidate, but we can imagine more clusters emerging if more ideological space existed between the two candidates.
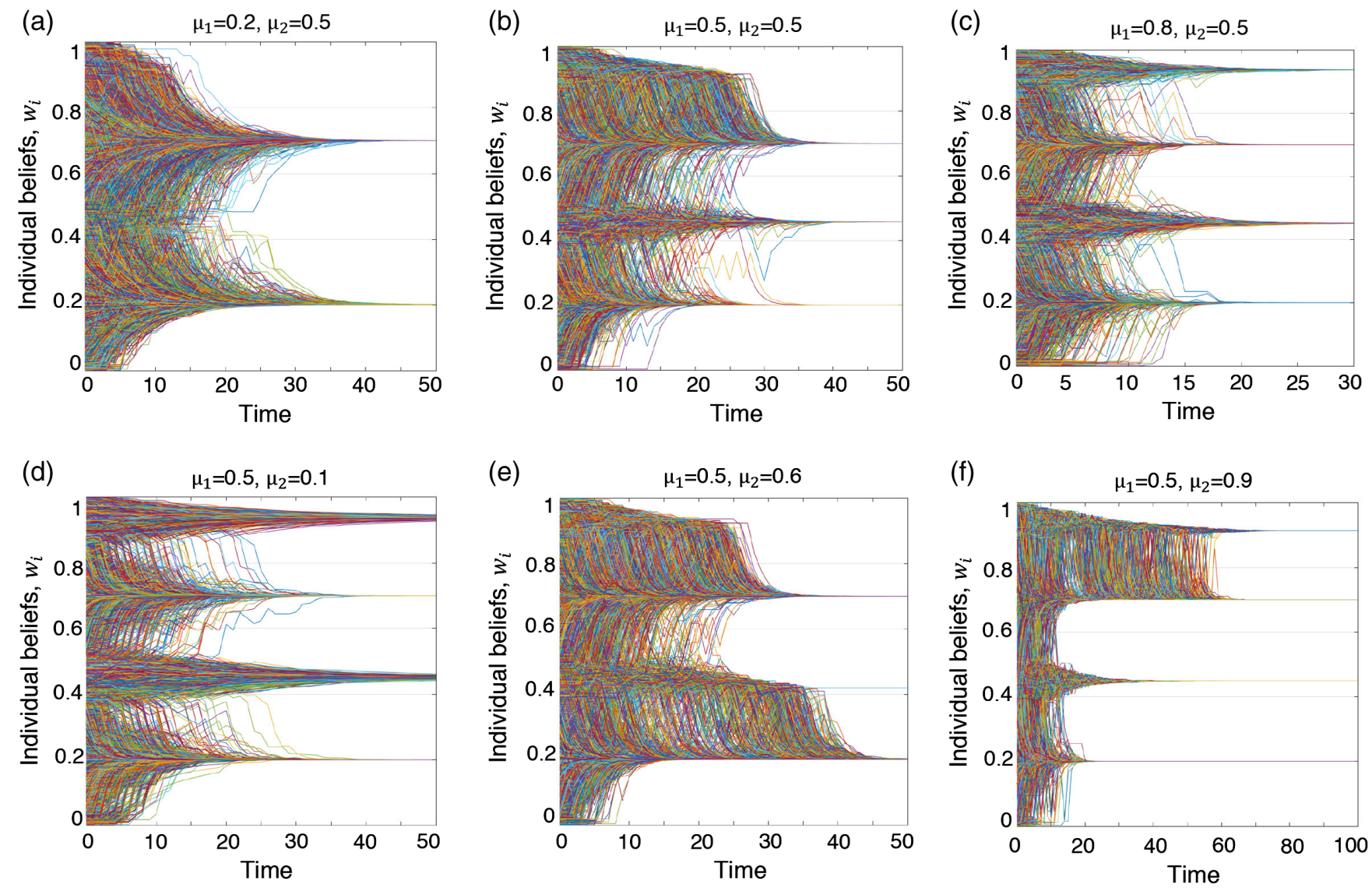

FIG. 4. Evolution of individual beliefs under different degrees of confirmation bias. (a)-(c) Strong positive feedback between candidates and voters accelerates the opinion polarizing process and leads to insufficient social discourse where more undecided individuals exist. (d)-(f) Intermediate levels of discussion partner influence increase interactions between individuals of different beliefs, which reduces the undecided people and effectively prevents the formation of small clusters of extremists. Parameters: All simulations begin from an ER graph with $N=10^{4},\langle k\rangle=6, \Omega_{T}=0.5, \theta_{1}=0.2, \theta_{2}=0.7$, and $\delta_{1}=0.18$. 
The stubbornness of individuals protects them from the influence of centralized sources of influence (campaigns), allowing the formation of independent echo chambers. At medium values $\left(0.19<\delta_{1}<0.43\right)$ of open-mindedness, all individuals congeal around one of the two candidates and seek out connections with like-minded people. Each echo chamber corresponds to a centralized campaign message that individuals either go to directly or eventually are pulled toward by their increasingly homophilous social networks. Individuals are polarized because they are just openminded enough to social influence for polarization to occur. As open-mindedness becomes high $\delta_{1}>0.43$, however, the population forms one giant cluster. Individuals are willing to consider a wide range of political messages, and initial ideology has a much weaker correlation with the ideology of the candidate that each individual chooses. In these environments, it would be expected that the relative efforts of the campaigns would be very important, as most individuals are open to most options. Individuals in this situation will not rewire their ties, and most political influence will happen between individuals and candidates. (The complete evolution of these three network topologies can be found in Movies S1-S3 [46].)

\section{B. Peer and candidate influence and ideological alignment}

We now turn our attention to how different sources of social influence interact to create echo chambers or preserve ideological diversity (Fig. 3). In our model, there are two parameters that govern social influence processes and the belief shifts of individuals: $\mu_{1}$ is the extent to which individual beliefs become aligned with those of their selected candidate, and $\mu_{2}$ is the extent to which individual beliefs become aligned with those of their trusted discussion partners. We would expect high levels of $\mu_{1}$ to correspond with convergence around candidate viewpoints and high levels of $\mu_{2}$ to lead to decentralized and emergent convergence around more arbitrary viewpoints. Intuitively, we would expect that high levels of either or both would lead to echo chambers and ideological homogeneity.
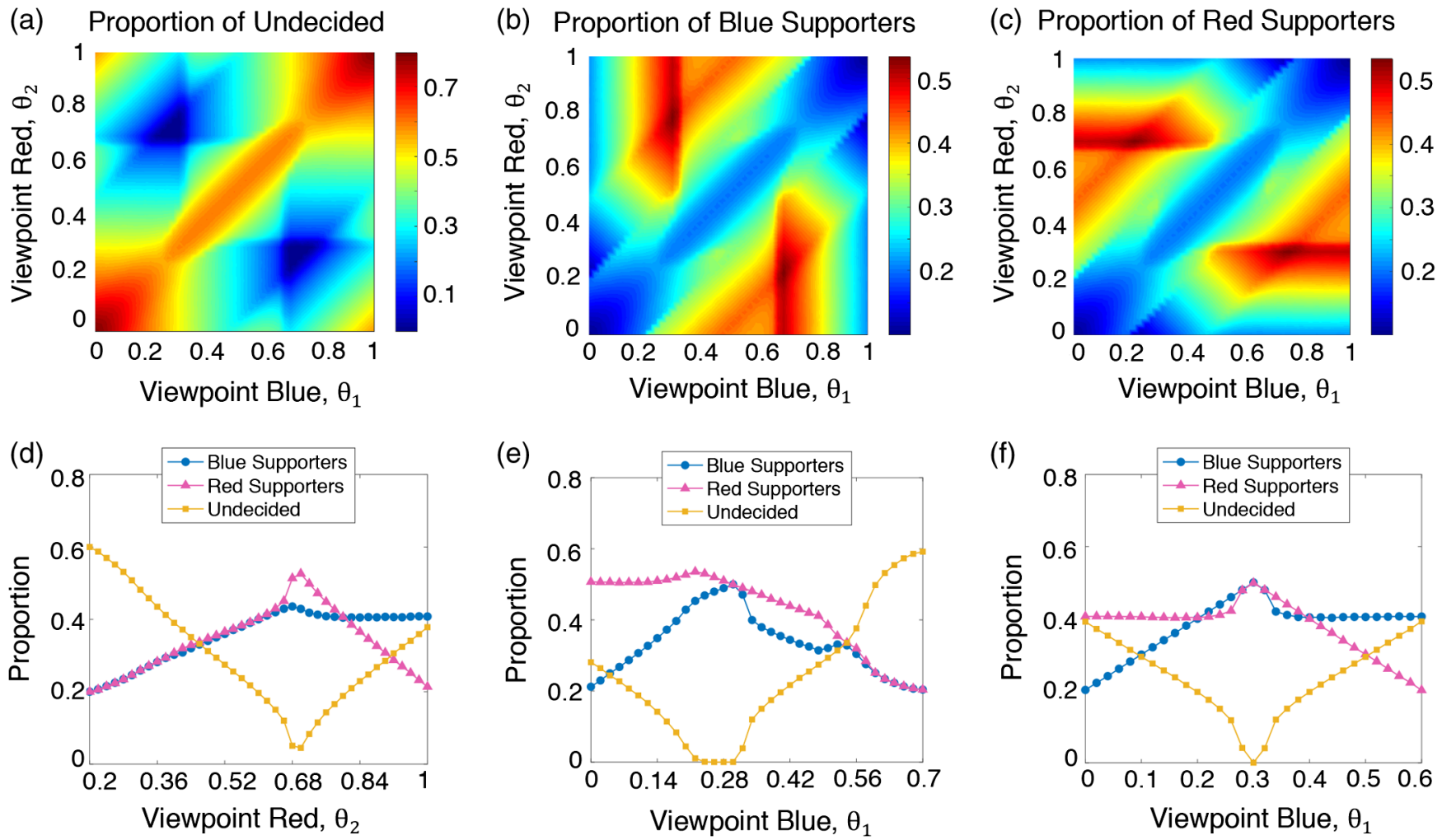

FIG. 5. Competing campaign ideologies within social networks. We can also use our model to see how campaigns should ideologically position themselves to maximize voter appeal. We assume that, in the short run, the open-mindedness (identity scope) of voters and levels of discussion partner and candidate influence on voters remains constant, so we fix $\delta_{1}=0.18$ and $\mu_{1}=\mu_{2}=0.5$. (a)-(c) show how vote shares for blue, red, and undecided varies as a function of the candidate's ideological positions. Both candidates maximize vote share by picking opposing positions in the ranges of [0.2, 0.3] and [0.7, 0.8]. In (d),(e), the ideological viewpoint of one campaign is fixed [(d) $\theta_{1}=0.2$ and (e) $\left.\theta_{2}=0.7\right]$ and the other is varied. In (f), the difference between the two campaigns is fixed $\left(\delta_{0}=0.4\right)$, and maximum vote share for both candidates occurs when the campaigns are equidistant from $0.5\left(\theta_{1}=0.3\right.$ and $\left.\theta_{2}=0.7\right)$. All simulations begin with an ER graph with $N=10^{4},\langle k\rangle=6$, and $\Omega_{T}=0.5$. Simulation results are averaged over 100 independent runs. 
The results of the model are less intuitive. The emergence of echo chambers and alignment with political candidates tends to be highest for moderate levels of peer influence and low levels of candidate influence [Figs. 3(a) and 3(b)]. Very high levels of candidate influence turn all supporters into extremists and leave candidate supporters beyond the ideological scope of their undecided peers [Figs. 3(c) and 3(e) and 4(a)-4(c)]. Low levels of both forms of influence similarly leave individuals outside the scope of candidates stranded in an undecided part of the ideological spectrum. Extremely high levels of peer influence may similarly leave certain people behind if their peers congeal around viewpoints too quickly. What seems to be most conducive to echo-chamber formation is a moderate process of peer influence that pulls people into camps slowly without leaving behind undecided stragglers, perhaps guided by very slight top-down influence from candidates [see the phase region where $\mu_{1} \in[0,0.3]$ and $\mu_{2} \in[0,0.2]$ in Fig. 3(a)]. High levels of social influence in a population where people are reluctant to hearing different beliefs can create many different ideological islands that people will be stuck on but may prevent the emergence of two dominant and opposing opinions [Figs. 3(d), 3(f), 4(d)-4(f)].

It is worth noting that our results are robust when the initial ideological distribution of agents is initially polarized. Two sets of simulations for a symmetrically and asymmetrically polarized population are included as robustness checks (Fig. 11; see details in Appendix C).

\section{The ideological positions and efforts of political campaigns}

In the presence of social dynamics that lead to the formation of echo chambers, it may be logical for political candidates to take on positions that are more extreme than what would predicted by standard median-voter models of political competition $[48,49]$. Given a fixed level of voter open-mindedness (identity scope), peer influence, and candidate influence, candidates may be incentivized to take on viewpoints that are more opposed to one another instead of converging around the median. In Figs. 5(a)-5(c), we can see that the best response of a candidate is not to simply position themselves immediately to the side of their opponent that is closer to the median but rather to stake out a position that is positioned in a large gap between their opponent and the opposite end of the political spectrum. Vote shares by viewpoint for different candidates in different scenarios are displayed in Figs. 5(d)-5(f); we see that red's best response to a blue viewpoint of 0.2 is around 0.68 [Fig. 5(d)], that blue's best response to a red viewpoint of 0.7 is around 0.3 [Fig. 5(e)], and that, given a fixed viewpoint distance between red and blue of 0.4 , both candidates maximize proportion of their support by aligning symmetrically around the median (0.5) and selecting viewpoints of 0.3 and 0.7 .
We also study the influence of campaign effort on winning majority support (Fig. 6). The importance of campaign effort in our model is largely dependent on the ratio of the identity scope $\left(\delta_{1}\right)$ of individuals to the spread of the candidate positions $\left(\delta_{0}\right)$. We denote this value as $\rho=\delta_{1} / \delta_{0}$. When $\rho=1 / 2$, by definition there are no voters ideologically between the two candidates who could possibly avoid the influence of either candidate, but in the absence of peer influence each fall in line with the candidate who is closest to them. Figure 6 shows how campaign effort varies in effectiveness as a function of $\rho$. As $\rho$ goes from 0 to $1 / 2$, the effects of relative campaign efforts may be trivial in the absence of large differences [Figs. 6(a) and 6(b)]. For levels of $\rho$ slightly lower than $1 / 2$, excessive campaigning may even be counterproductive if campaigns accidentally pull in potential supporters too quickly, leaving undecideds more vulnerable to their opponent [Fig. 6(c)]. As $\rho$ goes from $1 / 2$
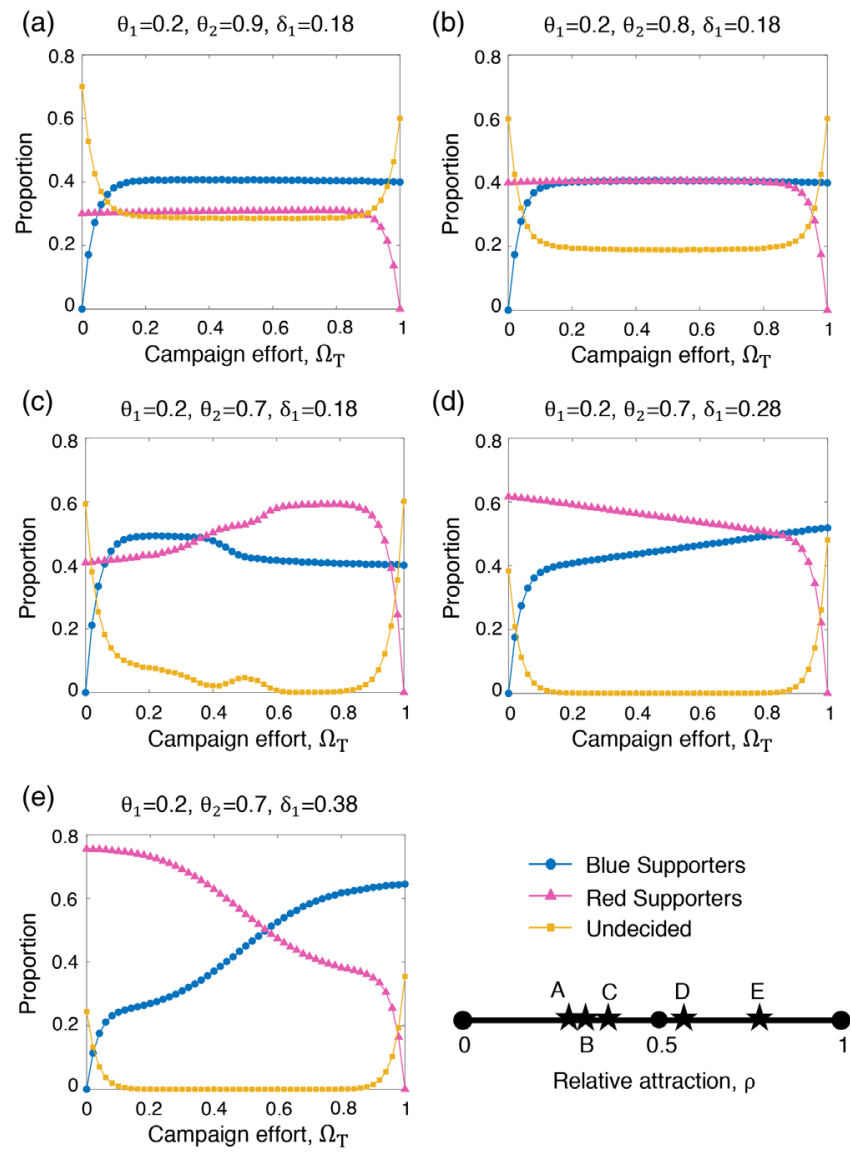

FIG. 6. Impact of campaign effort on winning majority support. (a)-(e) As the relative attraction of the viewpoints (defined as identity scope divided by candidate spread) in a campaign competition increases $(\rho=0.257,0.3,0.36,0.56$, and 0.76 , respectively), the relative efforts of campaigns become more important. In some cases, the effects of campaign effort become counterproductive [see (c)]. Parameters: All simulations begin from an ER graph with $N=10^{4},\langle k\rangle=6$, and $\mu_{1}=\mu_{2}=0.5$. Simulation results are averaged over 100 independent runs. 
(a)

First debate

Second debate

Third debate
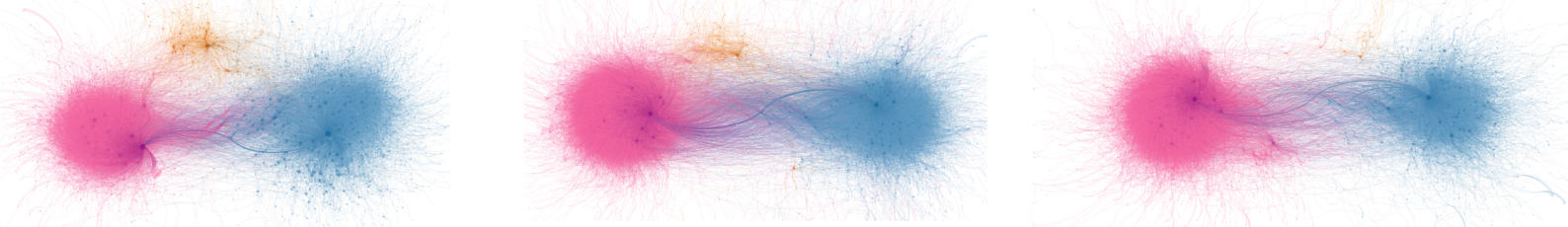

(b)

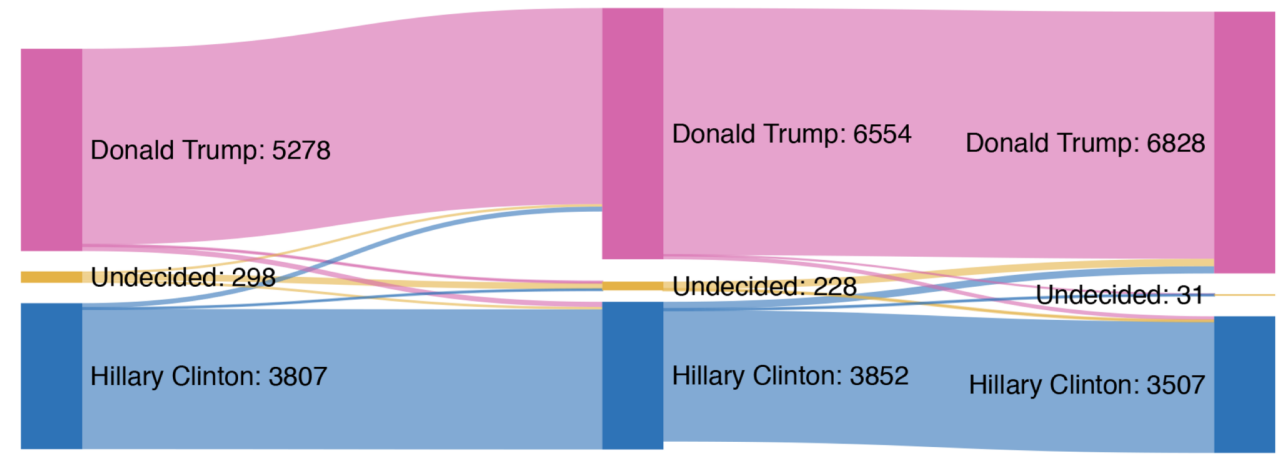

(c)
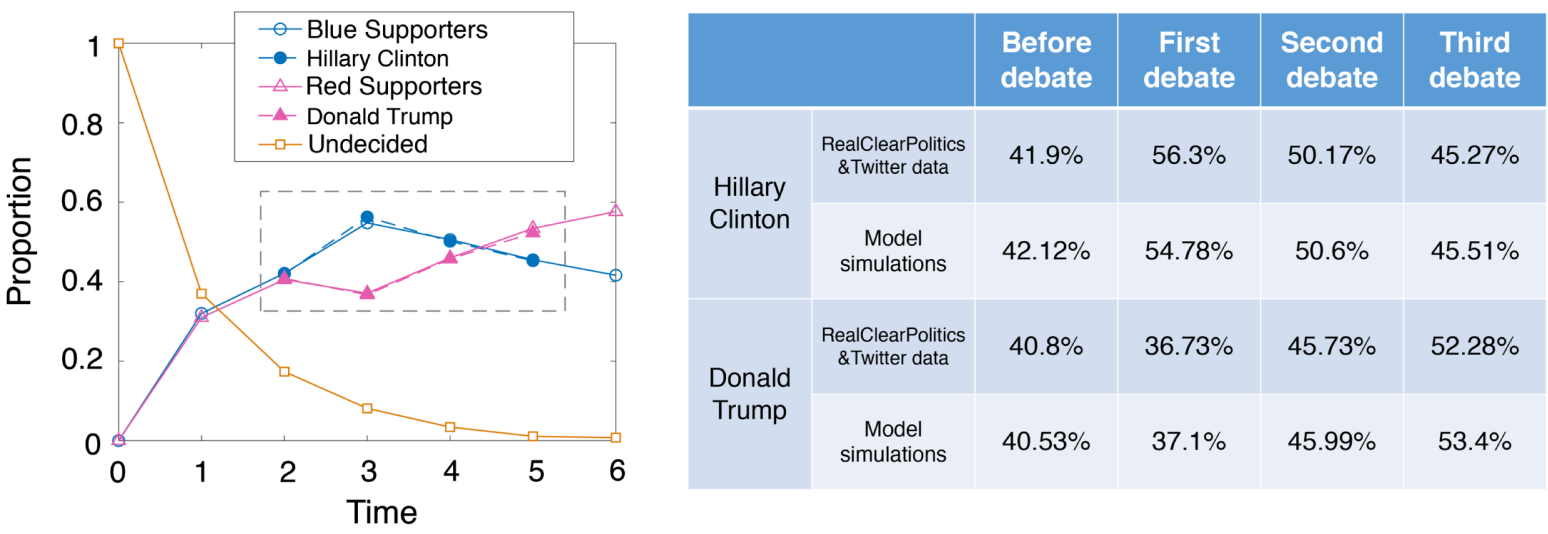

(d)

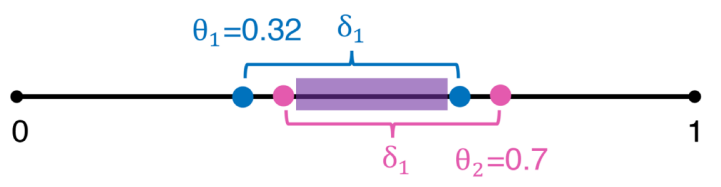

(1)

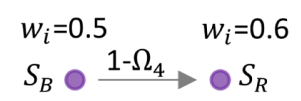

(2)

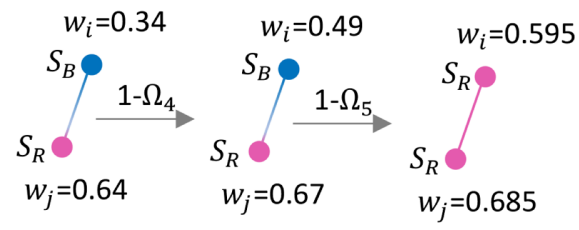

FIG. 7. Model-data integration provides insights into the 2016 U.S. presidential election. (a) Retweet networks of the first, second, and third presidential debates in the 2016 U.S. presidential election show the rise of echo chambers that gradually absorb undecided voters. (b) Sankey diagram of individual-level opinion evolution for Twitter users between the first and second debates and the second and third debates. Most Donald Trump or Hillary Clinton supporters are "loyal followers who would not change their political tendency from beginning to end." (c) Reproduction of opinion evolution processes in the 2016 U.S. presidential election using an agent-based model. We take the Real Clear Politics polling average on September 15, 2016, as an initial estimate of predebate support, while, from the first to third debates, we use the same Twitter data as in (a). Fixing a middle level of confirmation bias $\left(\mu_{1}=\mu_{2}=0.5\right.$ ), we find a group of bestfitting parameters for the ideological positions of campaigns, the identity scope (open-mindedness) of voters, and relative levels of campaign effort: $\theta_{1}=0.32, \theta_{2}=0.7, \delta_{1}=0.32, \Omega_{1}=\Omega_{2}=0.5, \Omega_{3}=0.8, \Omega_{4}=0.4$, and $\Omega_{5}=0.1$ (we let $\Omega_{6}=\Omega_{5}=0.1$ to model one extra time step). Each result in the figure is the average of 100 simulations. (d) Illustration of the potential mechanisms that lead to side switching within this model (in this case, from blue supporter to red supporter). Two possible ways are shown: (1) a blue supporter within ideological range of both candidates switches via one single update as a result of campaigning; (2) switch via multiple updates, a voter is not persuaded by the candidate initially but instead talks to a discussion partner within their range who is a red supporter and then updates their beliefs. In the next time step, the voter is reached by the red campaign again and is close enough to the red candidate to be persuaded. 
to 1 , however, the number of voters who could support either candidate increases, increasing the importance of campaign efforts [Figs. 6(d) and 6(e)]. We also see across all models that there are much higher numbers of undecideds if campaign efforts are lopsided, as the message of one candidate is effectively drowned out by their opponent.

Taken together, our results show that the conditions that lead to echo chambers can create political incentives that oppose our common-sense notions of how to win an election. Campaigns no longer benefit from converging to the center, as the median voter theorem predicts. Furthermore, in certain cases where candidates are not too close together or too far apart ideologically, campaign efforts can be counterproductive, as they may polarize individuals that would otherwise be useful in winning over moderates and undecideds. This insight is important, especially given the relative spending and outcome of the 2016 U.S. presidential election.

\section{Model-data integration of opinion evolution in the 2016 U.S. presidential election}

To illustrate how real-world cases could be expressed in terms of the model that we propose, we turn back to our earlier example of dynamic polarization on Twitter in the 2016 U.S. presidential campaign and integrate our proposed model with observed data (Fig. 7). This process of integration is not meant to suggest that our model can perfectly explain our empirical example. Rather, it is meant to illustrate how we can describe an observed polarization process in terms of our proposed model mechanisms and parameters. As the campaign unfolds, data from Twitter show that network polarization increases as echo chambers absorb undecided voters over time [Fig. 7(a)]. Looking at the trajectories of individual Twitter user retweet patterns [Fig. 7(b)], we see that most individuals who initially supported Clinton or Trump remain fairly constant over time, with Donald Trump winning over many undecideds between the second and third debates.

Using the proposed model in tandem with observed data, we can estimate the position and effectiveness of campaigns while holding the socio-cognitive mechanisms that sustain echo chambers constant. This estimation gives us a sense for the importance of "top-down" campaigning and ideological positioning. We also allow the model to estimate the "identity scope" parameter to calculate and account for the open-mindedness of voters. Figure 7(c) shows how our agent-based model of opinion evolution aligns with shifting levels of support for Clinton and Trump during the 2016 campaign. We assume that the ideological viewpoints of both campaigns $\left(\theta_{1}\right.$ and $\left.\theta_{2}\right)$ as well as the population identity scope $\left(\delta_{1}\right)$ are unchanged throughout the campaign. Simulations start from an Erdös-Rényi graph with $N=10^{4}$ and $\langle k\rangle=4.5$, which replicates the size and degree distribution of the observed retweet data. We set a moderate level of population confirmation bias $\left(\mu_{1}=\mu_{2}=0.5\right)$. We then find a group of best-fitting parameters for the ideological polarization of the candidates and the level of campaign effort at each of the time steps $\left(\theta_{1}=0.32, \theta_{2}=0.7, \delta_{1}=0.32\right.$, $\Omega_{1}=\Omega_{2}=0.5, \Omega_{3}=0.8, \Omega_{4}=0.4$, and $\left.\Omega_{5}=0.1\right)$ using least-squares minimization with a simulated annealing algorithm (see Appendix B).

While the socio-cognitive mechanisms that lead to political polarization should theoretically lead to races that are boring and predictable because so many have already made up their minds, these results reveal just how dynamic and unpredictable the final phases of the 2016 U.S. presidential campaign were, with Clinton and Trump rapidly trading advantages in relative campaigns. Both candidates had ideological positions that were estimated to be a moderate distance from the median of 0.5 , with the Clinton campaign being slightly closer $\theta_{1}=0.32$ and $\left.\theta_{2}=0.7\right)$. Furthermore, the large estimated identity scope of 0.32 places a large proportion of voters within ideological reach of both campaigns. The model also shows wide swings in the relative effort of both campaign, reflecting a campaign where high levels of spending and social media outreach, news reports, and debate performances could rapidly change each candidate's appeal to undecided voters.

The observed data also show that a small fraction of individuals may have switched camps during the election [Fig. 7(b)]. Figure 7(d) illustrates how this process could occur within our proposed model: A "blue supporter" with neutral beliefs that are within reach of both camps [left side in Fig. 7(d)] may be directly persuaded by the "red campaign" [Fig. 7(d)(1)]. Or, a supporter with "blue beliefs" may interact with a "red neighbor" and be brought to a spot within reach of the red campaign [Fig. 7(d)(2)]. Overall, this figure is an example of how open-mindedness can increase the importance of campaign efforts. While polarization and voter stubbornness in the face of new information is detested in modern politics, in these examples, it makes individuals immune from falling under the influence of high-spending campaigns.

\section{CONCLUSIONS AND DISCUSSIONS}

Several empirical studies from large-scale social networks have provided valuable insights into the emergence of echo chambers. In addition, many mathematical models have shown how different combinations of social mechanisms and distributions of individuals can lead to emergent polarization and echo-chamber formation in a network. Some work has studied how smaller social media campaigns may act as stubborn agents that exert influence without being influenced themselves [50,51], but these "campaign" agents as modeled still pale in comparison to large-scale political campaigns, and their level of influence is dependent on their position in the larger network [52]. To obtain a more accurate picture of emergent polarization and echo-chamber formation in the modern media climate, large centrally located campaigns (i.e., campaigns that 
are potentially connected to everyone) must be considered, along with their influence on microlevel interactions between connected individuals and the evolving network structure of those interactions. Our analysis offers a theoretically driven agent-based model that can estimate and account for the effect of these mechanisms using realworld data.

Interactions between human behavior and social systems are often nonlinear and complex, and the aggregate consequences of small changes in individual behavior can be counterintuitive. The model we present here is no exception. A surprising insight is that bipartisan echo chambers may result from moderate levels of open-mindedness, where people are susceptible to slightly different beliefs but not overwhelmingly different beliefs, as opposed to extreme closed-mindedness (which preserves ideological diversity) or extreme open-mindedness (which brings everyone together somewhere in the middle). This finding is inherently uncomfortable. The idea of voters being ideologically flexible in the face of new facts, opinions, and arguments, while still maintaining a set of core principles, seems essential to a functioning democracy. Yet this "bend but do not break" style of opinion updating may be the very thing that leads to polarization. A purely stubborn or conformist population may both be less susceptible to the emergence of two dominant and opposing echo chambers.

While the formation of echo chambers and the issue of political polarization is the primary focus of our model, our results also have practical implications for political campaign strategy. Our findings suggest a potential downside to aggressive campaigning from persuasive but extreme candidates: Supporters may become too radical for their relatively moderate friends. In some cases, campaigns and candidates may be better off by being less persuasive, as this strategy gives their supporters ideological room to make their candidate's case in everyday discourse. Candidates with relatively extremal positions may do well by instead adopting a "big tent strategy," where they win supporters without persuading them to change their ideological stance. In the age of social media, political strategists may need to think more about the order in which they are targeting people and their commitment to ideological positions. While "firing up the base" may be important early on for gathering donations, it may also create a rift between supporters and potential converts. "Preaching to the choir" may make it difficult to fill up the pews.

Social division along political lines has aroused great concern since the 2016 U.S. presidential election [9,5355]. But the emergence of echo chambers is not confined to elections. In the age of "fake news," determining the truth in everyday affairs has also become a collective decisionmaking process prone to interacting cognitive biases and social influence dynamics. The spread of misinformation on social networks threatens the foundations of democracy and often disrupts everyday life [56-58]. The insights generated from our model suggest not only several techniques for inhibiting the emergence of echo chambers, but also for how to develop more effective messaging strategies for import public health issues, such as the importance of vaccinations or the potential dangers of vaping. In these cases, the public has a strong interest in generating a single echo chamber inhabited by the right idea. Our models suggest that this generation would be best accomplished through moderate messaging that gradually pulls individuals with extreme antivaccine or provaping beliefs closer to the mainstream, as opposed to stronger messaging that may convert moderate skeptics but leave extremists isolated in a smaller echo chamber. Ultimately, what turns a set of likeminded individuals into an ideological bubble is the disappearance of other people who can come in and change their mind.

\section{ACKNOWLEDGMENTS}

We gratefully acknowledge David Rand, who provided constructive suggestions on examining another dynamical model (as we provided in Supplemental Material [46]) and adding individual-level observations in the 2016 U.S. presidential election. X. W.'s work is supported by Program of National Natural Science Foundation of China Grants No. 11871004 and No. 11922102 and National Key Research and Development Program of China Grant No. 2018AAA0101100. F. F. is supported by a Junior Faculty Fellowship awarded by the Dean of the Faculty at Dartmouth and also by the Bill \& Melinda Gates Foundation (Grant No. OPP1217336), the NIH Centers of Biomedical Research Excellence (COBRE) Program (Grant No. 1P20GM130454), the Neukom CompX Faculty Grant, the Dartmouth Faculty Startup Fund, and the Walter \& Constance Burke Research Initiation Award.

\section{APPENDIX A: TWITTER DATA PROCESSING}

All original Twitter datasets are publicly available [59]. The raw Twitter data are not allowed to be shared online according to Twitter's terms of service; therefore, all the datasets are Twitter IDs. We first download the tweets using Twitter's API and obtain user ID (nodes), retweeting relationship (edges), and the time stamp from each tweet. Computational constraints prevent us from plotting the whole network, so we use a random sample to obtain 10\%$20 \%$ data of the original datasets for the first, second, and third debates and then focus on the largest connected component (which contains over 90\% of nodes). Layouts are constructed using the Force Atlas 2 algorithm in GEPHI. Nodes are weighted by the number of times they are retweeted. To capture the core structural characteristics of opinion evolution without losing any information, we record all nodes weights and get rid of pure followers who are not being retweeted (in degree equal to 0 ) and retweet 


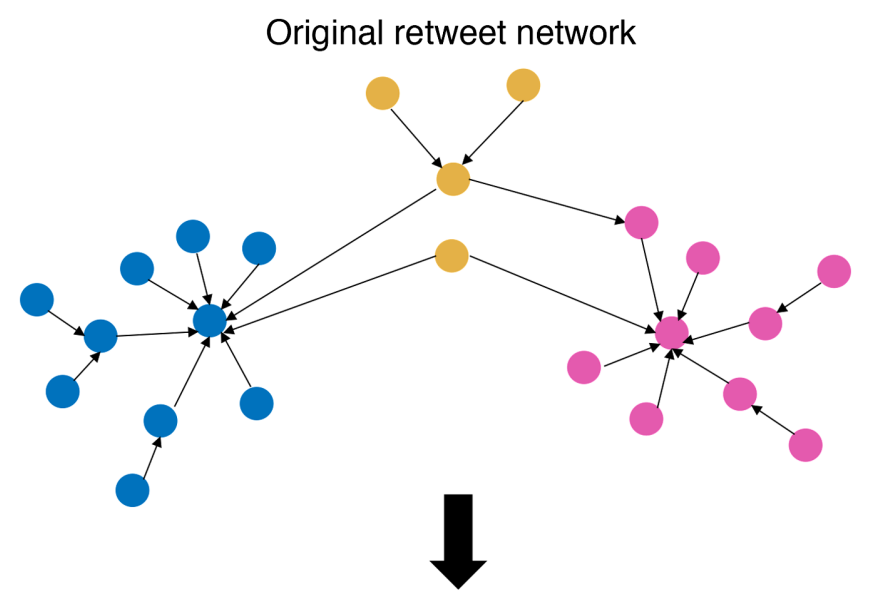

Core retweet network with weighted nodes

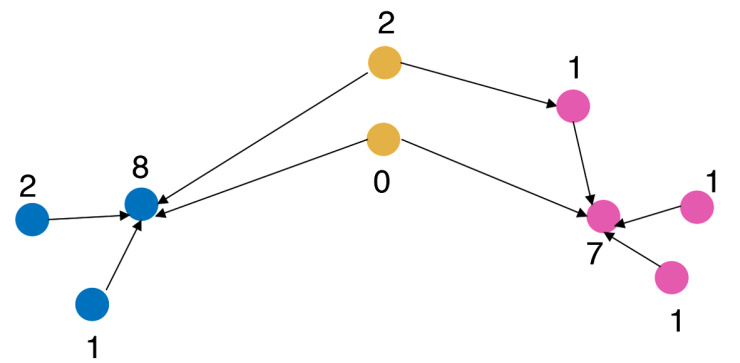

FIG. 8. Data-processing method of retweet networks. Define the weight of each node as the total number of times it is retweeted. In the largest connected component, we get rid of those "pure followers" who are not being retweeted (in degree equal to 0 ) and retweet others only once (out degree equal to 1) and plot the remaining retweet network with all nodes' weights recorded. The simplified retweet network has all the important information and better represents the core structural characters.

others only once (out degree equal to 1 ). This process leads to a more simplified retweet network with weighted nodes (Fig. 8). This simplification takes out about half of all edges and improves the precision of the community analysis. The retweet networks in Fig. 7(a) include 54840 edges and 25375 nodes for the first debate, 81055 edges and 33995 nodes for the second debate, and 58354 edges and 26219 nodes for the third debate. Finally, the weighted supporter estimates for each camp (or the supporter strength observed on social networks) can be approximately calculated by simply summing the weight of all nodes within each echo chamber. The sample datasets we use in this paper and the data-processing codes (written in PYTHON3) can be obtained at Ref. [60].

All the network figures in this paper use the Force Atlas 2 layout algorithm from GEPHI [61]. The color for each node is calculated using the Louvain algorithm for community detection [62].

We stress that our main focus is to understand how public opinion evolves through individual decision-making processes and socio-cognitive biases using agent-based models. Small inaccuracies in the true proportions of candidate supporters that are caused by random sampling and the community detection algorithm are unavoidable but tolerable for our purposes.

\section{APPENDIX B: DATA FITTING METHOD}

Within the time scope of a single event, such as the 2016 U.S. presidential election, we assume that the inherent psychological and cognitive properties (i.e., the identity scope and the effect of discussion partner and candidate influence) of Twitter users do not change by a discernable amount. Therefore, we aim to find a static set of values for $\theta_{1}, \theta_{2}, \delta_{1}, \mu_{1}$, and $\mu_{2}$ with a changing group of $\Omega_{T}$ that can best mimic the evolution of the campaign. The simulation results of four successive time steps (from $T=2$ to $T=5$ ) are expected to reproduce the real data. To reduce the computational complexity of the fitting procedure, we fix a moderate degree of candidate influence and discussion partner influence and set $\mu_{1}=\mu_{2}=0.5$. Other parameters are constrained as follows: $\theta_{1} \in[0,0.5], \theta_{2} \in[0.5,1]$, $\delta_{1} \in\left[0, \theta_{2}-\theta_{1}\right]$, and $\Omega_{T} \in[0,1], T \in\{1,2,3,4,5\}$. The step length for $\theta_{1}, \theta_{2}$, and $\delta_{1}$ is 0.02 , while the step length for $\Omega_{T}$ is 0.1 . We take the average value of 100 times simulations as the final results for each group of parameters. We develop a simple simulated annealing (SA) algorithm with least-squares minimization to find the best fit of real data, which is as follows:

(1) Initialization and definitions.-Define $S=\left\{\theta_{1}\right.$, $\left.\theta_{2}, \mu_{1}, \Omega_{T}\right\}$ as the set of all possible combinations of parameters. According to the descriptions of parameter range and step length above, $S$ is a finite set. Denote $R$ as the eight-dimensional vector of real data (containing the proportions of supporters for two candidates before debate and during the first, second, and third debates). Initially, the iteration counter $i=0$ and an initial state is set $S_{0} \in S$. In addition, we select a starting temperature $T_{0}$ and a freezing threshold $T_{\min }$ with a cooling rate $r$. At time step $i, S_{i}$ is the current state, and $T_{i}$ is the cooling schedule. Based on the least-squares minimization, we define the cost function $f\left(S_{i}\right)=\frac{1}{N} \cdot\left(\|\right.$ aver $\left.-R \|^{2} / \sigma^{2}\right)$, where $N=8$ is the dimension of $R$, aver is the average result (also a eight-dimensional vector) of 100 simulations for state $S_{i}, \|$ aver $-R \|$ is the Euclidean distance of vector aver and $R$, and $\sigma=1 \%$ is a precision control coefficient for the global fitting.

(2) The SA methods.-For time step $i+1$, a new state $\bar{S}$ is randomly generated from $S-\left\{S_{i}\right\}$, and the Markov chain of the state vector $S_{i}$ is determined by the following:

The algorithm allows prior information and can be recycled by setting $S_{0}$ as the best group of parameters that we have already found. The $\mathrm{C}++$ code for this simulated annealing algorithm along with our dynamic 
Algorithm 1: Simulated annealing.

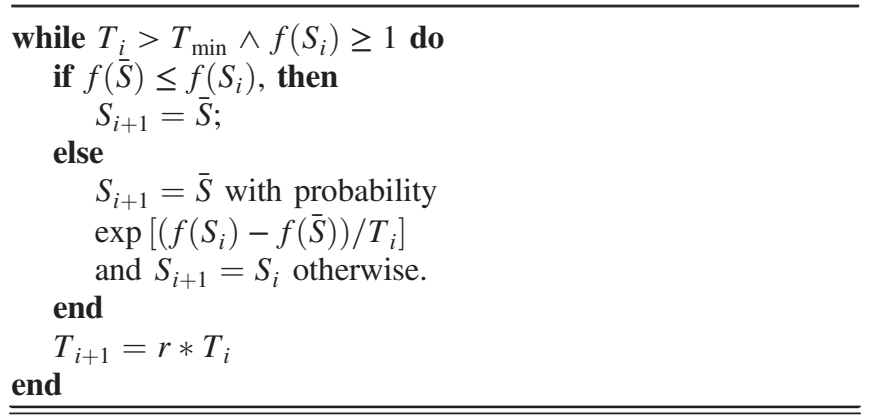

model is available [60]. The poll data before debate can be found in Ref. [63].

\section{APPENDIX C: MODEL ROBUSTNESS}

In our basic model, we assume both that there is a single level of individual open-mindedness shared by all agents and that there is a uniform distribution of initial beliefs along the interval from 0 to 1 . In this section, we relax these two assumptions to examine the robustness of our conclusions.

(1) Heterogeneity of open-mindedness. - Instead of uniform $\delta_{1}$, here we assume each individual $i$ has a unique value of open-mindedness $\delta_{1, i}$, drawn from a truncated normal distribution, which we believe more accurately reflects the distribution of what might be considered an individual personality or cognitive trait. Mathematically, $\delta_{1, i}$ has a normal distribution with mean $\delta_{1}^{\prime}$ and variance $\sigma^{2}$ and lies within the interval $[0,0.5]$. Results show that, as $\delta_{1}^{\prime}$ becomes larger, there are still three phase stages in the polarization process divided by two transition points (Fig. 9). Similar to our initial model, two opposing echo chambers are more likely to occur when population open-mindedness $\delta_{1, i}$ surrounds a moderate $\delta_{1}^{\prime}$. We do find, however, that a higher level
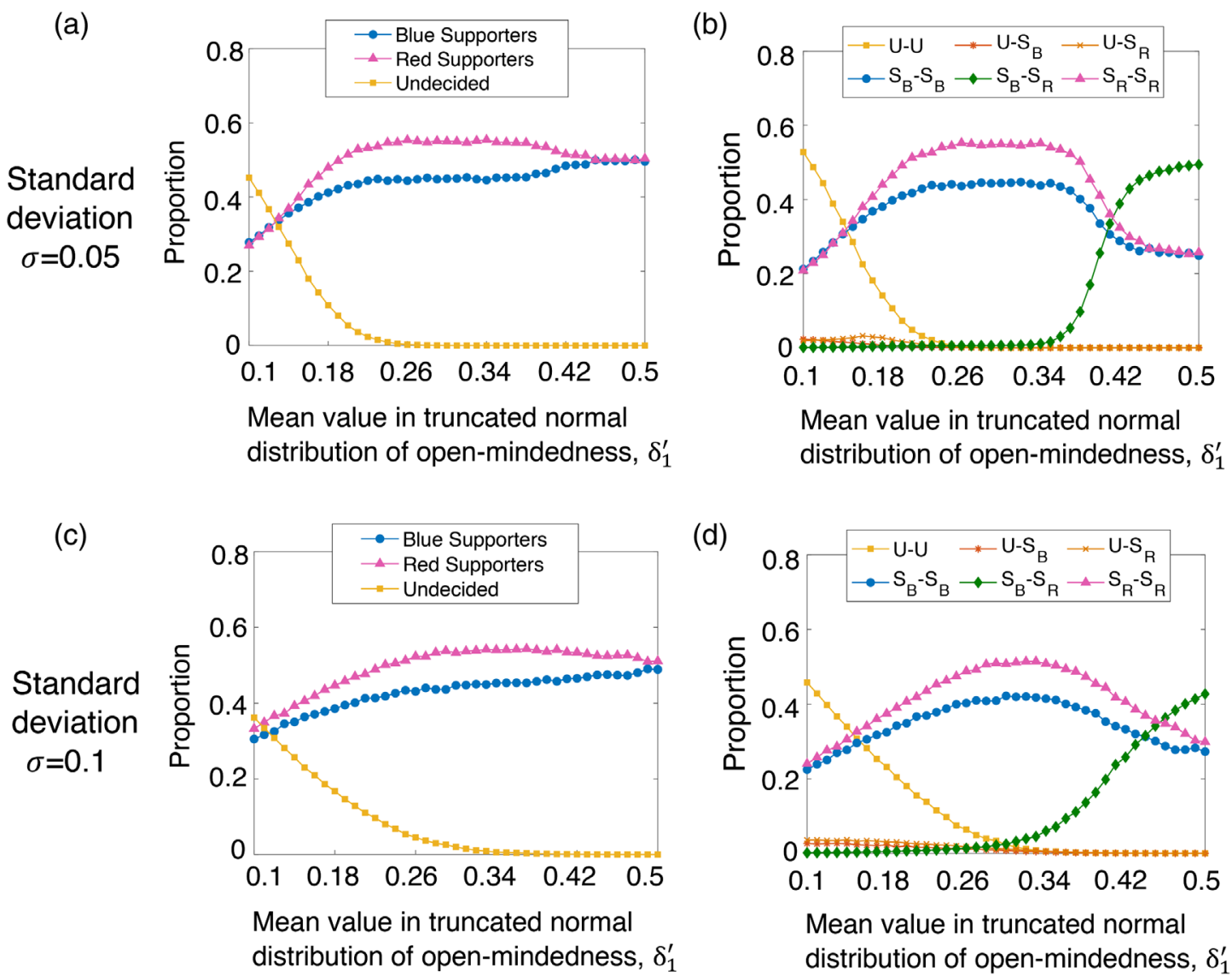

FIG. 9. Emergence of echo chambers with heterogenous levels of population open-mindedness. We relax the assumption of homogenous population open-mindedness $\left(\delta_{1}\right)$ and instead allow a truncated normal distribution of open-mindedness $\left(\delta_{1, i}\right)$. As $\delta_{1}^{\prime}$ increases, we observe (similar to the main model) that there are three phase stages divided by two transition points. Two opposing echo chambers are more likely to occur when individual open-mindedness $\delta_{1, i}$ surrounds a moderate $\delta_{1}^{\prime}$. Compared to mild heterogeneity (a),(b), strong heterogeneity allows for wavering individuals or independent clusters for slightly higher levels of mean open-mindedness (c),(d). Simulation results are averaged over 30 independent runs. Parameters: All simulations begin from an ER graph with $N=10^{4}$, $\langle k\rangle=6, \Omega_{T}=0.5, \theta_{1}=0.2, \theta_{2}=0.7, \mu_{1}=0.5$, and $\mu_{2}=0.5$. 
(a) Distribution of open-mindedness

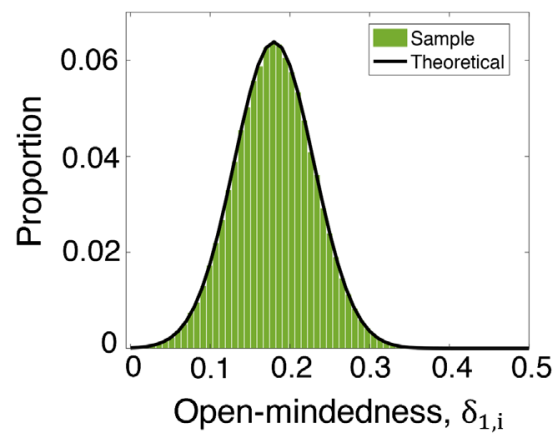

(d) Distribution of open-mindedness

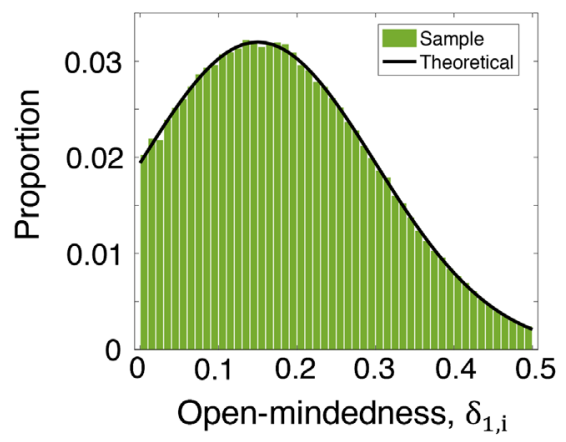

(b) Proportion of Undecided

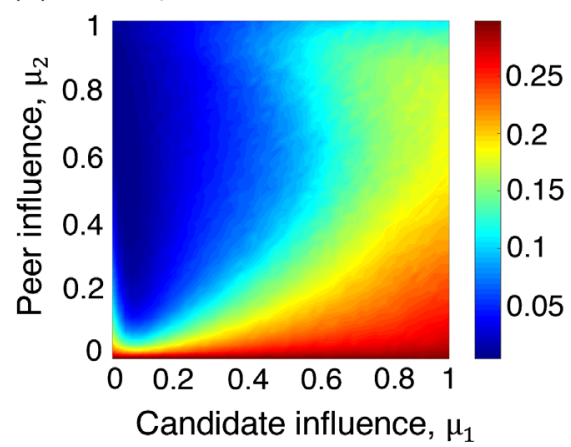

(e) Proportion of Undecided

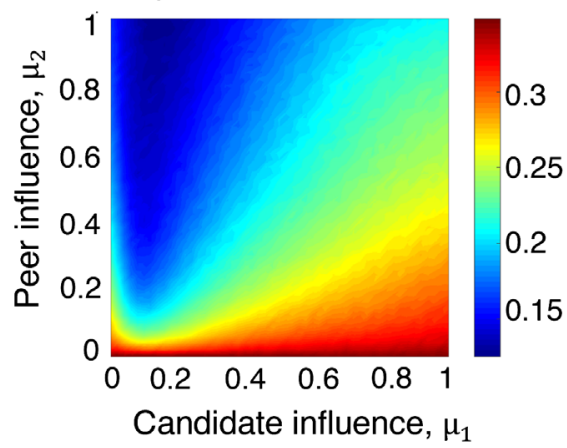

(c) Proportion of Red Supporters

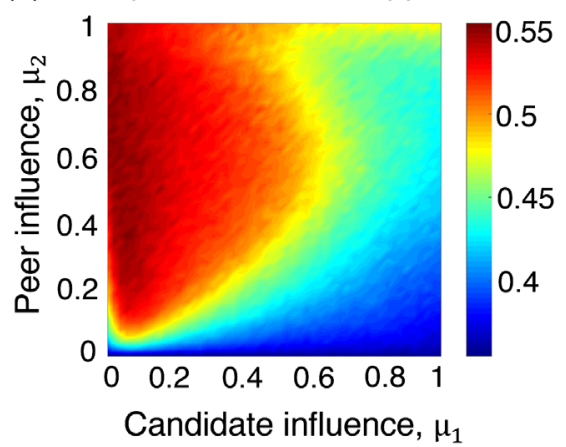

(f) Proportion of Red Supporters

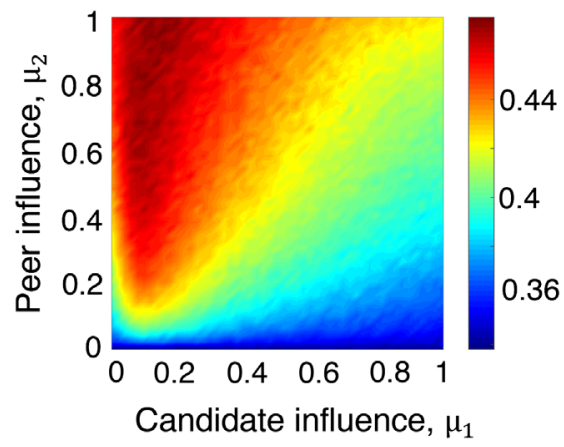

FIG. 10. Effect of candidate influence and peer influence on polarization under heterogenous population open-mindedness. We run simulations with mild heterogeneity in open-mindedness (a)-(c) and strong heterogeneity in open-mindedness (d)-(f). Higher levels of heterogeneity change the relationship between candidate influence, peer influence, and polarization. In higher heterogeneity and highpeer-influence conditions, candidate influence has less of a dampening effect on polarization. Furthermore, higher levels of heterogeneity generally lead to less polarization across all combinations of influence parameters. Simulation results are averaged over 30 independent runs. Parameters: All simulations begin from an ER graph with $N=10^{4},\langle k\rangle=6, \Omega_{T}=0.5, \theta_{1}=0.2$, and $\theta_{2}=0.7$.

of heterogeneity (larger standard deviation $\sigma$ ) in open-mindedness slightly blurs these transition points.

Furthermore, we find that, in the mild heterogeneity condition, low levels of candidate influence and moderate levels of peer influence best facilitate the formation of opposing echo chambers [Figs. 10(a)-10(c), $\delta_{1}^{\prime}=0.18$ and $\sigma=0.05$ ]. This finding is similar to the original (homogeneous) model, but in this case high levels of candidate influence do result in higher polarization when paired with high levels of peer influence. Simulations conducted with a higher level of heterogeneity [Figs. 10(d)-10(f), $\delta_{1}^{\prime}=0.15$ and $\sigma=0.15$ ] show similar but not identical patterns to the mild heterogeneity condition. In this case, the highest levels of polarization are seen with low levels of candidate influence and high levels of peer influence, but, once again, there is some polarization when peer influence and candidate influence are both high. However, across all values of peer and candidate influence, polarization is higher in the mild heterogeneity condition than in the strong heterogeneity condition.

(2) Polarized distribution of initial beliefs. - Intuitively, the influence of peer and candidate influence and the impacts of an external political campaign should depend on the initial distribution of political beliefs. We conduct two sets of simulations where the initial distribution is polarized, where we assume $w_{i}$ follows bimodal distributions. In each set of simulations, the distribution is bimodal: The probability density function (PDF) can be expressed as $f\left(w_{i}\right)=$ $0.5 * \operatorname{norm}\left(w_{i}, w_{1}^{\prime}, \sigma_{1}\right)+0.5 * \operatorname{norm}\left(w_{i}, w_{2}^{\prime}, \sigma_{2}\right)$, where $\operatorname{norm}\left(w_{i}, w^{\prime}, \sigma\right)$ reflects the PDF of the normal distribution with mean $w^{\prime}$ and standard deviation $\sigma$, evaluated at value $w_{i}$. In addition, $w_{i}$ is confined to $[0,1]$. We consider two types of polarized distribution: one symmetrical and one asymmetrical. In the symmetrical condition, $w_{1}^{\prime}=0.2, \sigma_{1}=0.15$ and $w_{2}^{\prime}=0.8, \sigma_{2}=0.15$ [Figs. 11(a)-11(c), 12(a)-12(c), and $13(\mathrm{a})-13(\mathrm{c})]$. In the asymmetrical condition, 
$w_{1}^{\prime}=0.15, \sigma_{1}=0.15 \quad$ and $\quad w_{2}^{\prime}=0.8, \sigma_{2}=0.25$

[Figs. 11(d)-11(f), 12(d)-12(f), and 13(d)-13(f)].

While the polarized conditions, not surprisingly, have higher levels of polarization overall (fewer undecideds), the simulations from the main text otherwise appear to be nearly identical to the simulations that involve bimodal initial distributions. In each set of simulations, low levels of candidate influence and middling levels of peer influence seem to be most conducive to polarizing supporters (Fig. 11). The effect of polarized initial distributions appears independent of the effect of peer influence and candidate influence. In addition, results with regard to competing campaign ideologies and campaign effort also largely resemble findings from the original model: Candidates collectively capture more voters and minimize undecideds by having opposing views (Fig. 12); too much or too little campaign effort can leave certain individuals undecided (Fig. 13).

In summary, our main conclusions remain largely consistent when we relax two main assumptions by (i) allowing cognitive heterogeneity in terms of agent open-mindedness or (ii) initializing the simulation with a prepolarized agent electorate. The only notable differences are that high levels of open-mindedness heterogeneity slightly alter the relationship between peer influence, candidate influence, and polarization and that higher levels of heterogeneity can decrease polarization across the board.

\section{APPENDIX D: REVIEW OF PREVIOUS MODELS ON ECHO CHAMBERS AND OPINION EVOLUTION}

A canonical set of social influence models with regards to a continuously valued attribute, as opposed to the diffusion of a binary variable, were proposed by Friedkin and Johnsen to understand attitude change in small groups. These models allow for peer influence between connected actors, while individuals still retain a variable level of their initial beliefs, attitudes, or opinions $[34,35]$. More complex social influence models incorporate several other features. The opinion or belief being updated may be multidimensional [36]. The rules dictating belief updates by individuals may go beyond simple diffusion from one agent to another: Rules might involve logical constraints [37], multiple individuals might update their opinions simultaneously, or influence may be a function of prior ideological similarity [38,39]. The arrow from connection to influence may be reversed, with ties between similar but disconnected agents forming and ties between dissimilar individuals fading $[40,41]$. (a) Polarized distribution of initial beliefs

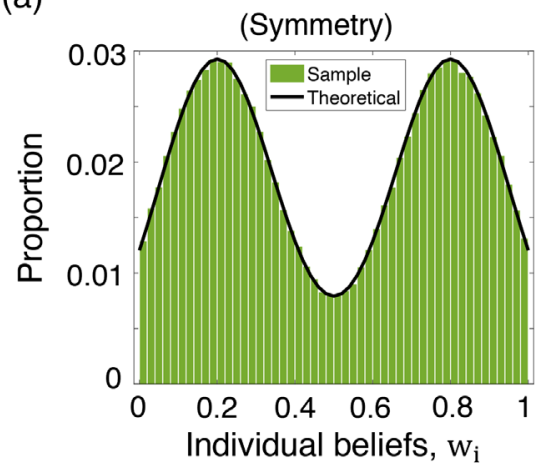

(d) Polarized distribution of initial beliefs

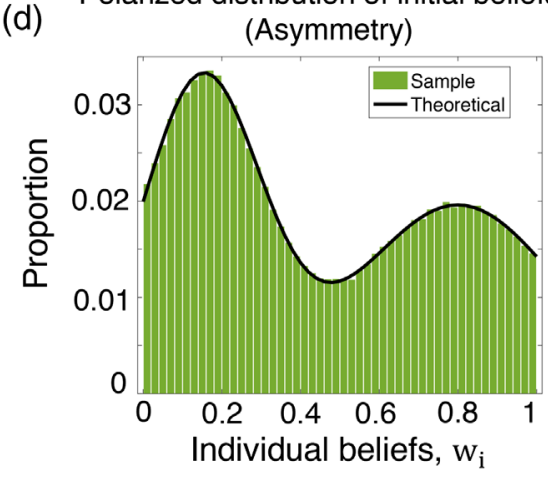

(b)

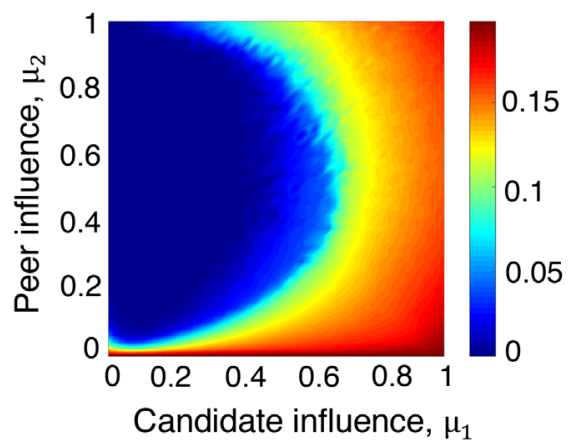

(e) Proportion of Undecided

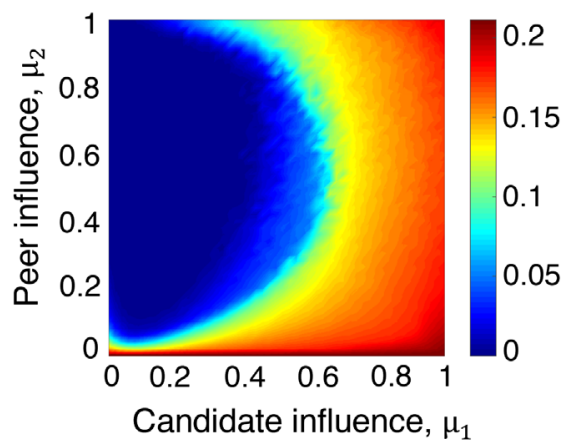

(c) Proportion of Red Supporters

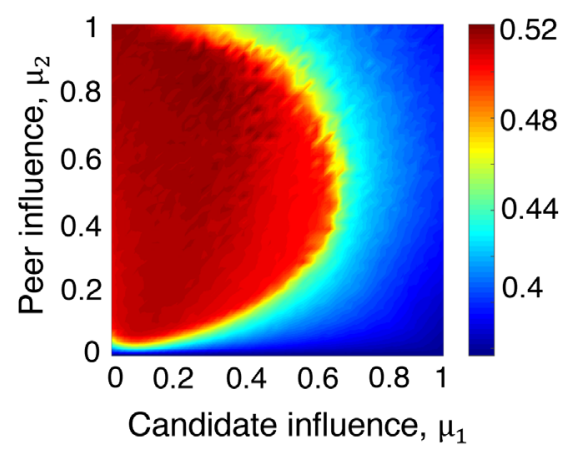

(f) Proportion of Red Supporters

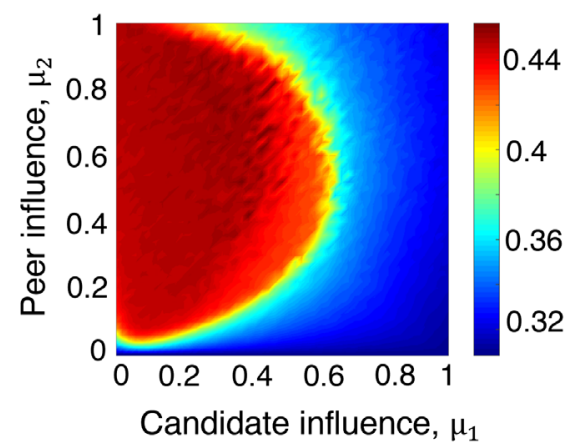

FIG. 11. Candidate influence and peer influence under polarized initial beliefs. We relax the initial condition of individual beliefs from uniform distribution to (a)-(c) symmetrical bimodal distribution and (d)-(f) asymmetrical bimodal distribution. Simulation results are averaged over 30 independent runs. Parameters: All simulations begin from an ER graph with $N=10^{4},\langle k\rangle=6, \Omega_{T}=0.5, \theta_{1}=0.2$, $\theta_{2}=0.7$, and $\delta_{1}=0.18$. 
These basic social mechanisms at the individual level can lead to noteworthy results at the population level. The combination of homophily (tie rewiring) and influence can lead to polarization [40], uniform opinion [41], and the correlation of otherwise totally unrelated opinions, attitudes, or preferences [50]. Activists who seek to spread extreme opinions, or stubborn agents who remain inflexible, also influence macrolevel attitude distributions [32,51].

Of particular concern in recent years is the emergence of echo chambers $[31,45]$, which refers to the tendency from people with similar beliefs to speak to and hear from only one another, and can sustain and exacerbate processes of political polarization. Echo chambers, being an inevitable epiphenomenon of emergent polarization, emerge from rewiring and selective peer influence [42] and may be particularly strong when they align with controversial topics [43].

\section{APPENDIX E: NOVELTY AND CONTRIBUTIONS OF OUR APPROACH}

The importance of social connections and peer influence to the overall distribution of opinions and attitudes in a population cannot be overstated. However, in real life, these emergent processes are often guided by large groups of people who are well coordinated and well funded, have stable strategic or moral intentions, and can influence people far and wide. In the context of our model, these are political campaigns, although in a different context we may just as easily point to larger media conglomerate or a well-organized social movement. Our model incorporates these powerful voices into a model of influence that draws on Deffuant's bounded confidence model [39] and the tie rewiring processes that underlie other dynamic models of influence and connection [41]. We do this incorporation specifically through the following model specifications.

\section{Symmetrical polarized distribution of initial beliefs}
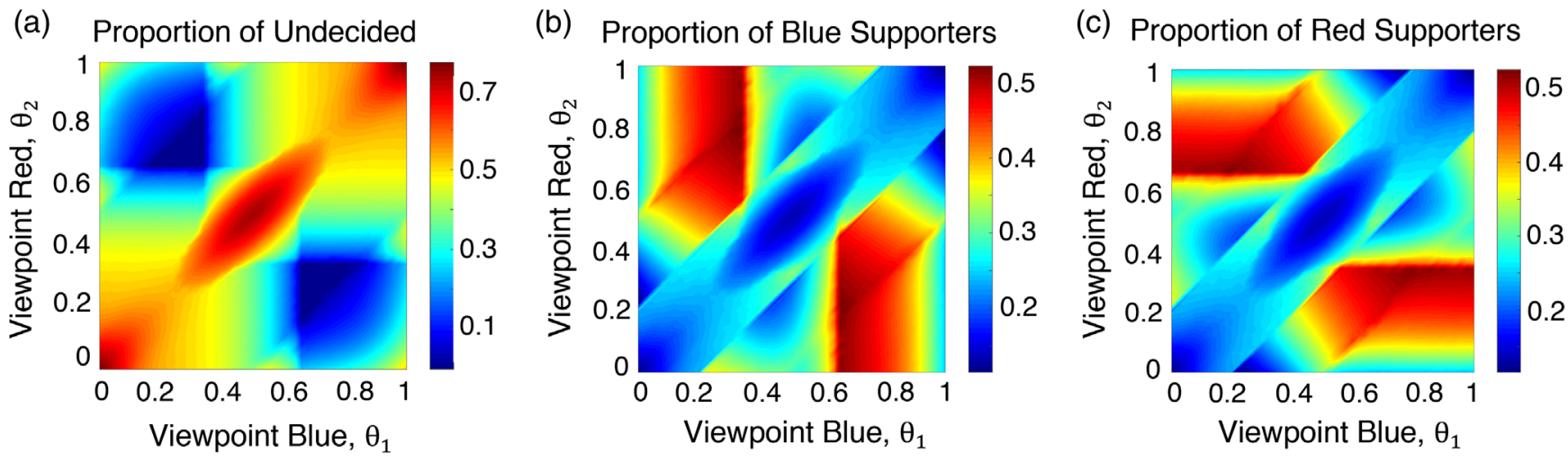

Asymmetrical polarized distribution of initial beliefs
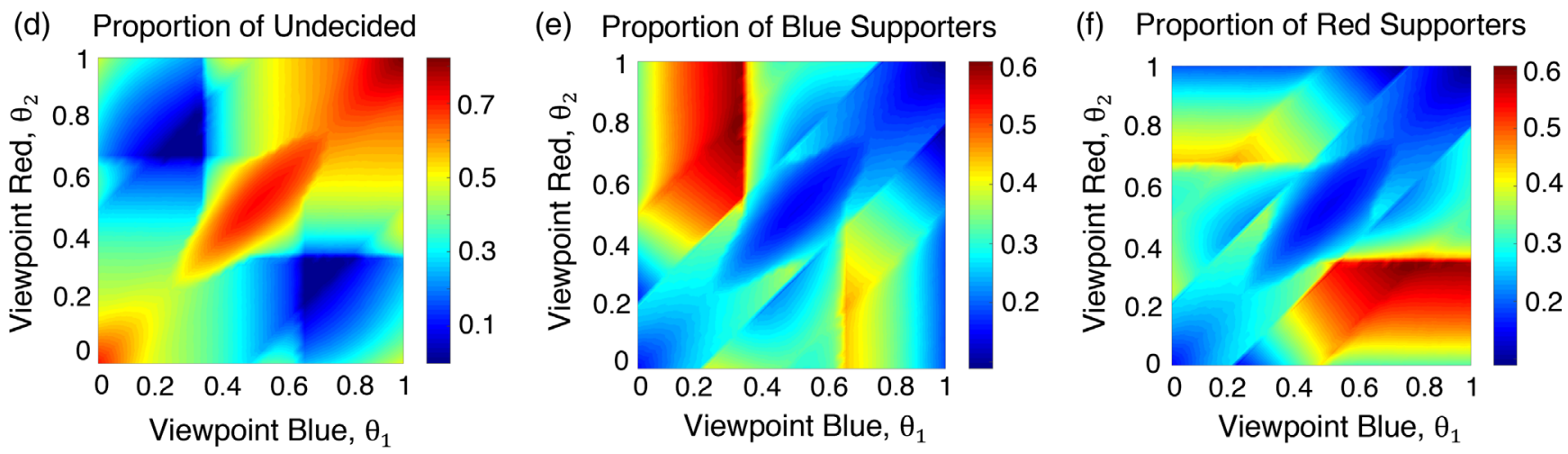

FIG. 12. Competing campaign ideologies under polarized initial beliefs. The initial condition of individual beliefs is relaxed from a uniform distribution to a symmetrical bimodal distribution (a)-(c) and an asymmetrical bimodal distribution (d)-(f). Results largely resemble findings from the original model: Candidates collectively capture more voters and minimize undecideds by having opposing views. Simulation results are averaged over 30 independent runs. Parameters: All simulations begin from an ER graph with $N=10^{4}$, $\langle k\rangle=6, \Omega_{T}=0.5, \delta_{1}=0.18, \mu_{1}=0.5$, and $\mu_{2}=0.5$. 
(1) Taking into account two layers of opinion or attitude dynamics [one's own opinion $\left(w_{i}\right)$ and whether or not to support A or B or neither in public discourse], our work explicitly characterizes the complex interplay between external political campaigns and opinion dynamics that occur between individual agents. We characterize political campaigns in terms of their ideological positions $\left(\theta_{1}\right.$ and $\left.\theta_{2}\right)$ and relative effort or effectiveness to one another over time $\left(\Omega_{T}\right)$. We also characterize individuals in terms of their susceptibility to campaign influence $\left(\mu_{1}\right)$ and the influence of their social connections $\left(\mu_{2}\right)$. To our knowledge, previous work does not account for the effect of central agents (political campaigns) that can influence all (noncampaign) agents in a dynamic model of influence and social connection.

(2) The incorporation of campaign influences and their positioning allows us to examine competitive campaign optimization (Sec. II.C). This framework provides a new perspective not only for political strategists, but for individuals interested in engineering more effective campaigns that deal with public health issues or social problems, such as motivating compliance with vaccinations or the adoption of proenvironmental behaviors [64].

(3) Whereas other models of influence and social connection find the existence of either one or two large homogenous clusters, our model has conditions which allow for the emergence of either "undecided" clusters or wavering individuals. We provide two schematics that illustrate the emergence of undecided or wavering individuals under the framework of top-down external political campaigns and interpersonal interactions in Figs. 14 and 15.

Moreover, we stress that there are fundamental differences between external political campaigns and

\section{Symmetrical polarized distribution of initial beliefs}
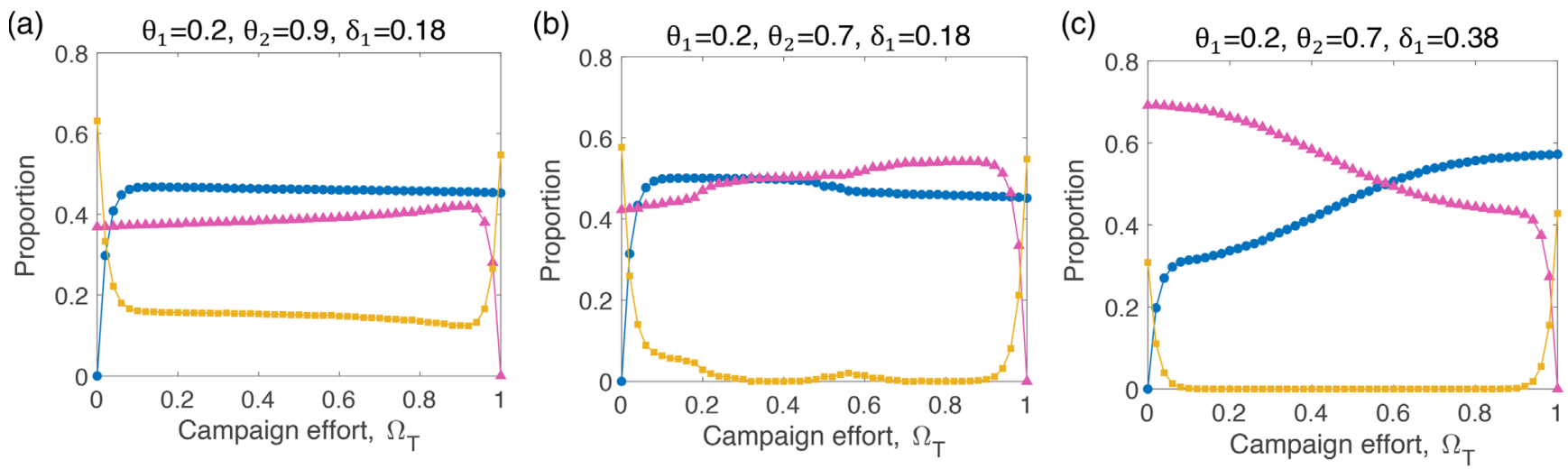

\section{Asymmetrical polarized distribution of initial beliefs}
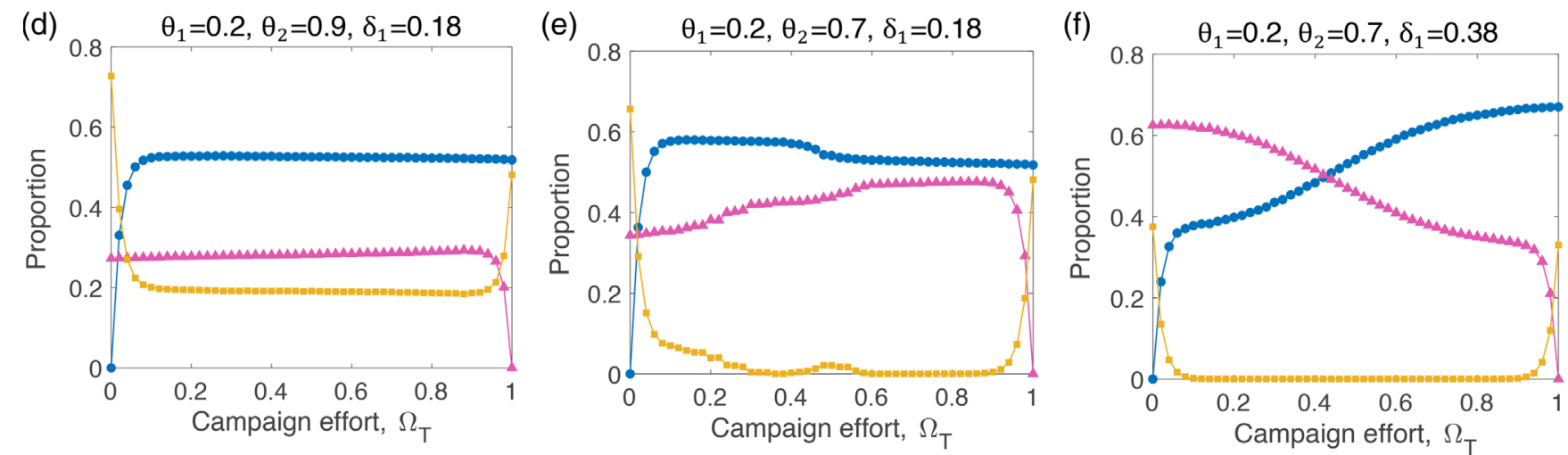

$\multimap$ Blue Supporters $\quad \longrightarrow$ Red Supporters $\quad \longrightarrow \quad$ - Undecided

FIG. 13. Impact of campaign effort on winning majority support under polarized initial beliefs. The initial distribution of individual beliefs is relaxed from uniform to a symmetrical bimodal distribution (a)-(c) and an asymmetrical bimodal distribution (d)-(f). The obvious finding that campaigns can suffer if they exert no effort, along with the counterintuitive finding that campaigning slightly too much can also slightly hurt their campaign, both replicate from the original model. Simulation results are averaged over 30 independent runs. Parameters: All simulations begin from an ER graph with $N=10^{4},\langle k\rangle=6, \theta_{1}=0.2, \theta_{2}=0.7, \delta_{1}=0.18, \mu_{1}=0.5$, and $\mu_{2}=0.5$. 


\section{Emergence of Undecided individuals}

(a)

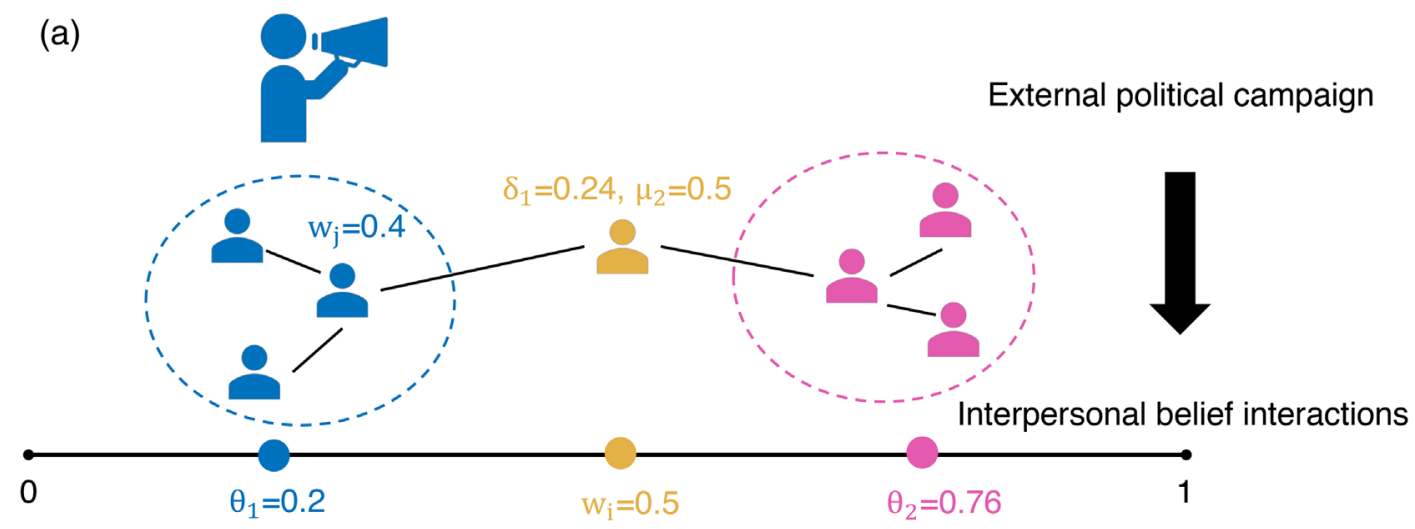

(b)

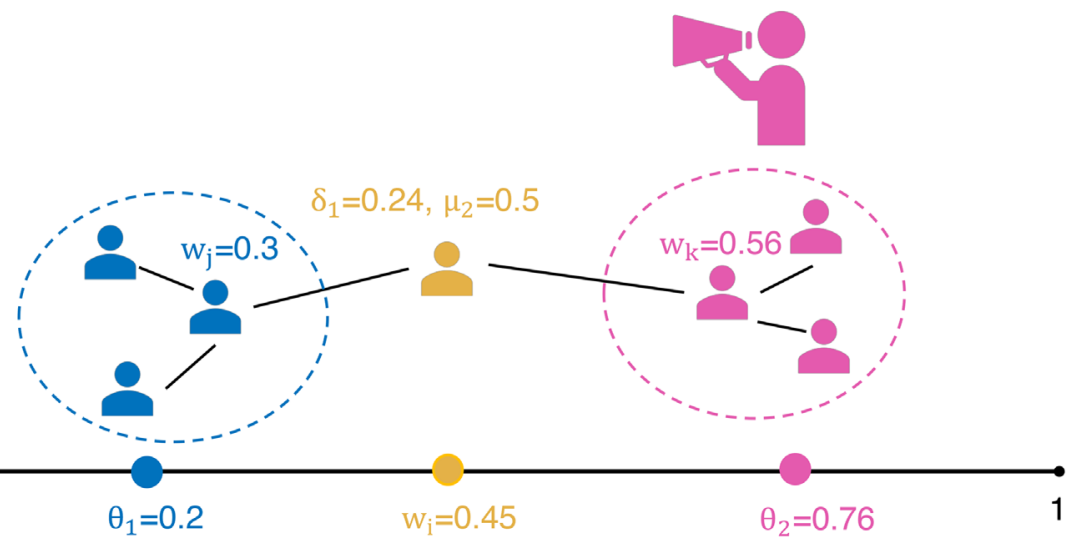

(c)

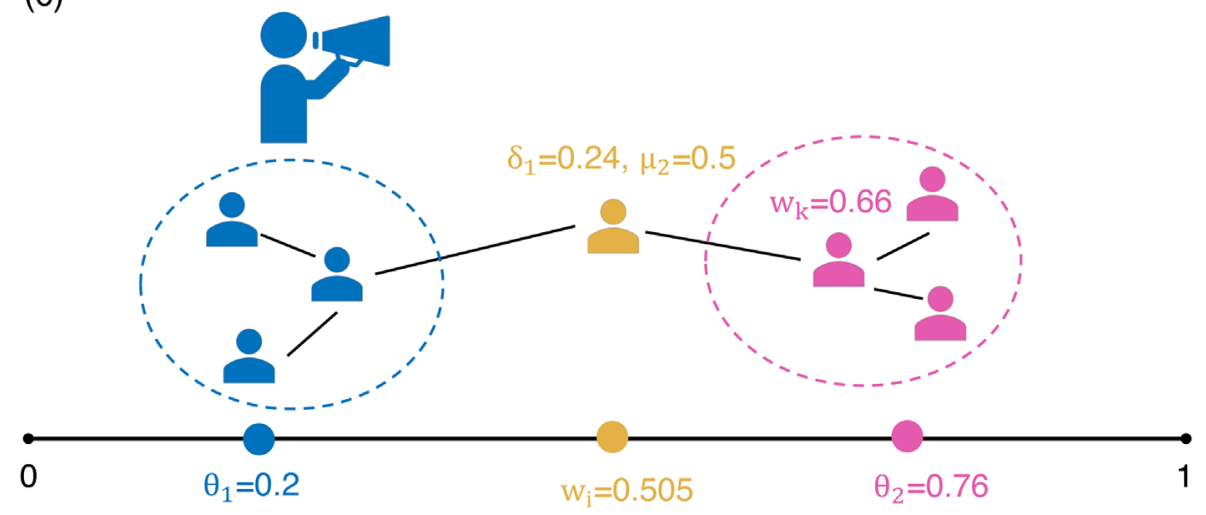

FIG. 14. Emergence of undecided individuals. (a),(b) The undecided individual $i$ updates with viewpoint blue $\left(\theta_{1}\right)$ that is beyond its identity scope; therefore, $i$ seeks friend $j$ for interpersonal interaction. (b),(c) The undecided individual $i$ updates with viewpoint red $\left(\theta_{2}\right)$ that is also beyond its identity scope and further discusses with friend $k$.

stubborn agents, which are shown to be important ingredients of polarization an echo-chamber formation in previous work [52]. The importance of stubborn agents is dependent on their initial position in the larger network, whereas political campaigns are inherently central actors in the network, as they can broadcast influence anyone. We believe this difference makes the model of opinion formation and social influence more realistic for largescale influence processes (such as national political campaigns). 


\section{Emergence of wavering supporters}

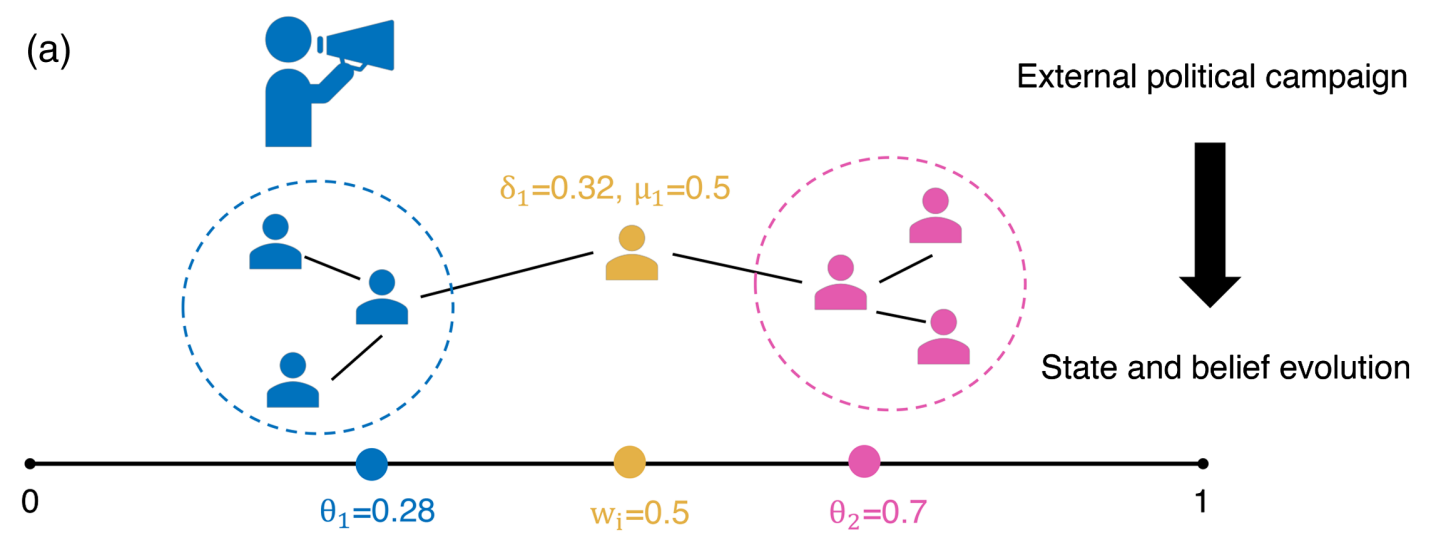

(b)
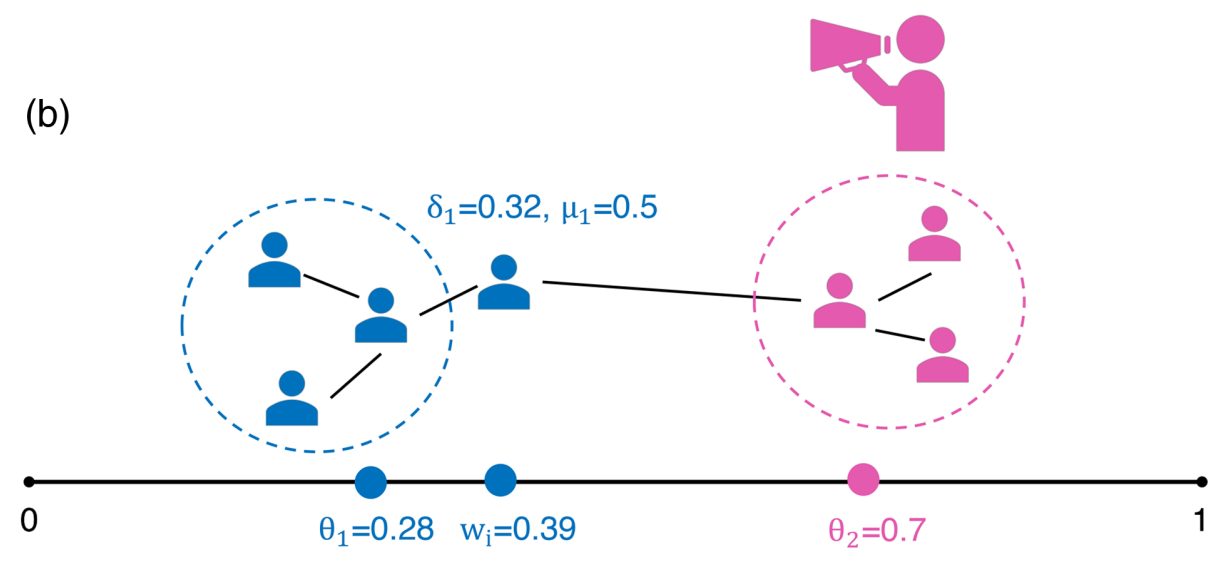

(c)

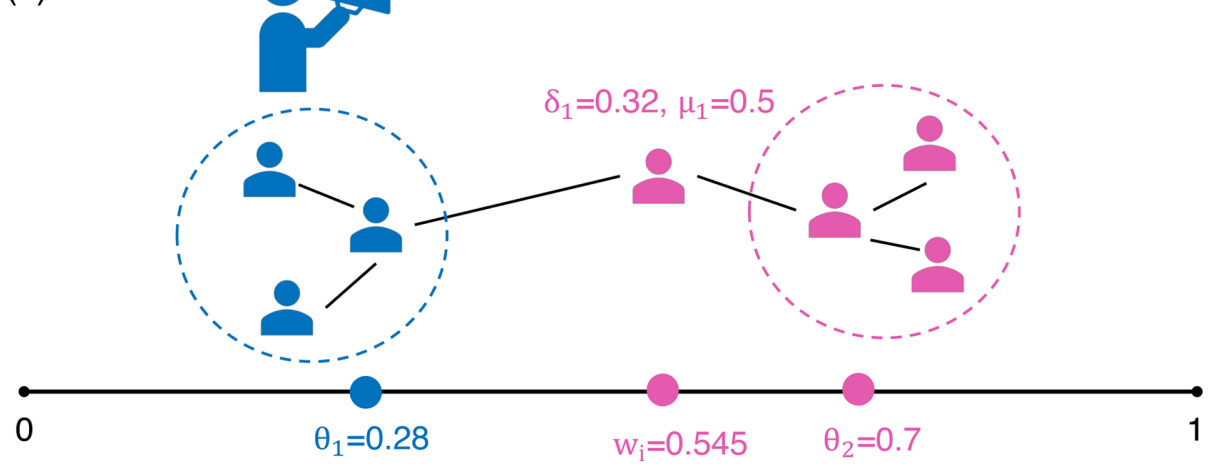

FIG. 15. Emergence of wavering supporters. (a),(b) The undecided individual $i$ updates with viewpoint blue and directly becomes a blue supporter with its belief $w_{i}$ converging to $\theta_{1}$. (b),(c) Blue supporter $i$ updates with viewpoint red and directly becomes a red supporter with its belief $w_{i}$ converging to $\theta_{2}$. 
[1] S. Plous, The Psychology of Judgment and Decision Making (McGraw-Hill, New York, 1993).

[2] R.S. Nickerson, Confirmation Bias: A Ubiquitous Phenomenon in Many Guises, Rev. Gen. Psychol. 2, 175 (1998).

[3] E. Jonas, S. Schulz-Hardt, D. Frey, and N. Thelen, Confirmation Bias in Sequential Information Search after Preliminary Decisions: An Expansion of Dissonance Theoretical Research on Selective Exposure to Information, J. Pers. Soc. Psychol. 80, 557 (2001).

[4] R. K. Garrett, Echo Chambers Online?: Politically Motivated Selective Exposure among Internet News Users, J. Computer-Mediated Commun. 14, 265 (2009).

[5] A. Bessi, M. Coletto, G. A. Davidescu, A. Scala, G. Caldarelli, and W. Quattrociocchi, Science vs Conspiracy: Collective Narratives in the Age of Misinformation, PLoS One 10, e0118093 (2015).

[6] N. Grinberg, K. Joseph, L. Friedland, B. Swire-Thompson, and D. Lazer, Fake News on Twitter during the 2016 US Presidential Election, Science 363, 374 (2019).

[7] K. Lewis, M. Gonzalez, and J. Kaufman, Social Selection and Peer Influence in an Online Social Network, Proc. Natl. Acad. Sci. U.S.A. 109, 68 (2012).

[8] J.-P. Onnela and F. Reed-Tsochas, Spontaneous Emergence of Social Influence in Online Systems, Proc. Natl. Acad. Sci. U.S.A. 107, 18375 (2010).

[9] H. Allcott and M. Gentzkow, Social Media and Fake News in the 2016 Election, J. Econ. Perspect. 31, 211 (2017).

[10] X. Wang, W. Li, L. Liu, S. Pei, S. Tang, and Z. Zheng, Promoting Information Diffusion through Interlayer Recovery Processes in Multiplex Networks, Phys. Rev. E 96, 032304 (2017).

[11] J. Kleinberg, Analysis of Large-Scale Social and Information Networks, Phil. Trans. R. Soc. A 371, 20120378 (2013).

[12] W. Quattrociocchi, R. Conte, and E. Lodi, Opinions Manipulation: Media, Power and Gossip, Adv. Complex Syst. 14, 567 (2011).

[13] J. Ugander, L. Backstrom, C. Marlow, and J. Kleinberg, Structural Diversity in Social Contagion, Proc. Natl. Acad. Sci. U.S.A. 109, 5962 (2012).

[14] D. Lazer, The Rise of the Social Algorithm, Science 348, 1090 (2015).

[15] E. Bakshy, S. Messing, and L. A. Adamic, Exposure to Ideologically Diverse News and Opinion on Facebook, Science 348, 1130 (2015).

[16] C. Castellano, S. Fortunato, and V. Loreto, Statistical Physics of Social Dynamics, Rev. Mod. Phys. 81, 591 (2009).

[17] R. M. Bond, C. J. Fariss, J. J. Jones, A. D. Kramer, C. Marlow, J. E. Settle, and J. H. Fowler, A 61-Million-Person Experiment in Social Influence and Political Mobilization, Nature (London) 489, 295 (2012).

[18] L. Liu, X. Wang, Y. Zheng, W. Fang, S. Tang, and Z. Zheng, Homogeneity Trend on Social Networks Changes Evolutionary Advantage in Competitive Information Diffusion, New J. Phys. 22, 013019 (2020).

[19] C. Wardle and H. Derakhshan, Information Disorder: Toward an Interdisciplinary Framework for Research and Policymaking, Council of Europe report, DGI, Vol. 9, 2017.
[20] K. Garimella, G. De Francisci Morales, A. Gionis, and M. Mathioudakis, Political Discourse on Social Media: Echo Chambers, Gatekeepers, and the Price of Bipartisanship, in Proceedings of the 2018 World Wide Web Conference on World Wide Web (International World Wide Web Conferences Steering Committee, Republic and Canton of Geneva, Switzerland, 2018), pp. 913-922.

[21] E. Colleoni, A. Rozza, and A. Arvidsson, Echo Chamber or Public Sphere? Predicting Political Orientation and Measuring Political Homophily in Twitter Using Big Data, J. Commun. 64, 317 (2014).

[22] A. Friggeri, L. Adamic, D. Eckles, and J. Cheng, Rumor Cascades, in Proceedings of the Eighth International AAAI Conference on Weblogs and Social Media (Association for the Advancement of Artificial Intelligence, AAAI, 2014), pp. 101-110.

[23] P. Metaxas, Evidence of Pizzagate Conspiracy Theory on Twittertrails, https://blogs.wellesley.edu/twittertrails/.

[24] T. Evans and F. Fu, Opinion Formation on Dynamic Networks: Identifying Conditions for the Emergence of Partisan Echo Chambers, R. Soc. Open Sci. 5, 181122 (2018).

[25] F. Fu and L. Wang, Coevolutionary Dynamics of Opinions and Networks: From Diversity to Uniformity, Phys. Rev. E 78, 016104 (2008).

[26] F. Zollo, P. K. Novak, M. Del Vicario, A. Bessi, I. Mozetič, A. Scala, G. Caldarelli, and W. Quattrociocchi, Emotional Dynamics in the Age of Misinformation, PLoS One 10, e0138740 (2015).

[27] J. G. Webster and T. B. Ksiazek, The Dynamics of Audience Fragmentation: Public Attention in an Age of Digital Media, J. Commun. 62, 39 (2012).

[28] A. Bessi, F. Zollo, M. Del Vicario, A. Scala, G. Caldarelli, and W. Quattrociocchi, Trend of Narratives in the Age of Misinformation, PLoS One 10, e0134641 (2015).

[29] D. Mocanu, L. Rossi, Q. Zhang, M. Karsai, and W. Quattrociocchi, Collective Attention in the Age of (Mis) Information, Comput. Human Behav. 51, 1198 (2015).

[30] S. Vosoughi, D. Roy, and S. Aral, The Spread of True and False News Online, Science 359, 1146 (2018).

[31] A. L. Schmidt, F. Zollo, M. Del Vicario, A. Bessi, A. Scala, G. Caldarelli, H.E. Stanley, and W. Quattrociocchi, Anatomy of News Consumption on Facebook, Proc. Natl. Acad. Sci. U.S.A. 114, 3035 (2017).

[32] D. DellaPosta, Y. Shi, and M. Macy, Why Do Liberals Drink Lattes?, Am. J. Sociology 120, 1473 (2015).

[33] A. Goldberg and S. K. Stein, Beyond Social Contagion: Associative Diffusion and the Emergence of Cultural Variation, Am. Sociol. Rev. 83, 897 (2018).

[34] N.E. Friedkin, A Structural Theory of Social Influence (Cambridge University Press, Cambridge, England, 2006), Vol. 13.

[35] N. E. Friedkin and E. C. Johnsen, Social Influence Network Theory: A Sociological Examination of Small Group Dynamics (Cambridge University Press, Cambridge, England, 2011), Vol. 33.

[36] S. E. Parsegov, A. V. Proskurnikov, R. Tempo, and N. E. Friedkin, Novel Multidimensional Models of Opinion Dynamics in Social Networks, IEEE Trans. Autom. Control 62, 2270 (2017). 
[37] N. E. Friedkin, A. V. Proskurnikov, R. Tempo, and S. E. Parsegov, Network Science on Belief System Dynamics under Logic Constraints, Science 354, 321 (2016).

[38] R. Axelrod, The Dissemination of Culture: A Model with Local Convergence and Global Polarization, J. Conflict Resolution 41, 203 (1997).

[39] G. Deffuant, D. Neau, F. Amblard, and G. Weisbuch, Mixing Beliefs among Interacting Agents, Adv. Complex Syst. 03, 87 (2000).

[40] M. Del Vicario, A. Scala, G. Caldarelli, H. E. Stanley, and W. Quattrociocchi, Modeling Confirmation Bias and Polarization, Sci. Rep. 7, 40391 (2017).

[41] P. Holme and M. E. J. Newman, Nonequilibrium Phase Transition in the Coevolution of Networks and Opinions, Phys. Rev. E 74, 056108 (2006).

[42] K. Sasahara, W. Chen, H. Peng, G. L. Ciampaglia, A. Flammini, and F. Menczer, On the Inevitability of Online Echo Chambers, arXiv:1905.03919 [J. Computat. Soc. Sci. (to be published)].

[43] F. Baumann, P. Lorenz-Spreen, I. M. Sokolov, and M. Starnini, Modeling Echo Chambers and Polarization Dynamics in Social Networks, Phys. Rev. Lett. 124, 048301 (2020).

[44] P. Barberá, J. T. Jost, J. Nagler, J. A. Tucker, and R. Bonneau, Tweeting from Left to Right: Is Online Political Communication More than an Echo Chamber?, Psychol. Sci. 26, 1531 (2015).

[45] M. Del Vicario, A. Bessi, F. Zollo, F. Petroni, A. Scala, G. Caldarelli, H. E. Stanley, and W. Quattrociocchi, The Spreading of Misinformation Online, Proc. Natl. Acad. Sci. U.S.A. 113, 554 (2016).

[46] See Supplemental Material at http://link.aps.org/ supplemental/10.1103/PhysRevX.10.041042 for Figs. S1S7 and Movies S1-S3: time evolutions of the three network topologies shown in Figs. 2(c)-2(e).

[47] J. Lorenz, Continuous Opinion Dynamics under Bounded Confidence: A Survey, Int. J. Mod. Phys. C 18, 1819 (2007).

[48] H. Hotelling, Stability in Competition, in The Collected Economics Articles of Harold Hotelling (Springer, New York, 1990), pp. 50-63.

[49] A. Downs, An Economic Theory of Democracy (Harper, New York, 1957).
[50] S. Galam and F. Jacobs, The Role of Inflexible Minorities in the Breaking of Democratic Opinion Dynamics, Physica (Amsterdam) 381A, 366 (2007).

[51] L. Böttcher, P. Montealegre, E. Goles, and H. Gersbach, Competing Activists Political Polarization, Physica (Amsterdam) 545A, 123713 (2020).

[52] D. Centola, J. Becker, D. Brackbill, and A. Baronchelli, Experimental Evidence for Tipping Points in Social Convention, Science 360, 1116 (2018).

[53] C. B. Williams and G. J. Gulati, Digital Advertising Expenditures in the 2016 Presidential Election, Social Science Computer Review 36, 406 (2018).

[54] A. Kucharski, Post-Truth: Study Epidemiology of Fake News, Nature (London) 540, 525 (2016).

[55] A. Bovet and H. A. Makse, Influence of Fake News in Twitter during the 2016 US Presidential Election, Nat. Commun. 10, 7 (2019).

[56] G. Pennycook and D. G. Rand, Fighting Misinformation on Social Media Using Crowdsourced Judgments of News Source Quality, Proc. Natl. Acad. Sci. U.S.A. 116, 2521 (2019).

[57] P. Williamson, Take the Time and Effort to Correct Misinformation, Nature (London) 540, 171 (2016).

[58] D. M. Lazer, M. A. Baum, Y. Benkler, A. J. Berinsky, K. M. Greenhill, F. Menczer, M. J. Metzger, B. Nyhan, G. Pennycook, D. Rothschild et al., The Science of Fake News, Science 359, 1094 (2018).

[59] https://www.docnow.io/catalog.

[60] https://github.com/fufeng/Public-discourse-and-social-networkecho-chambers.

[61] M. Jacomy, T. Venturini, S. Heymann, and M. Bastian, Forceatlas2, a Continuous Graph Layout Algorithm for Handy Network Visualization Designed for the GEPHI Software, PLoS One 9, e98679 (2014).

[62] V.D. Blondel, J.-L. Guillaume, R. Lambiotte, and E. Lefebvre, Fast Unfolding of Communities in Large Networks, J. Stat. Mech. (2008) P10008.

[63] https://www.realclearpolitics.com/epolls/2016/president/us/ general_election_trump_vs_clinton_vs_johnson_vs_stein5952.html.

[64] N. Johnson, R. Leahy, N. J. Restrepo, N. Velasquez, M. Zheng, P. Manrique, P. Devkota, and S. Wuchty, Hidden Resilience and Adaptive Dynamics of the Global Online Hate Ecology, Nature (London) 573, 261 (2019). 